Article

\title{
Physicochemical Insight into Coordination Systems Obtained from Copper(II) Bromoacetate and 1,10-Phenanthroline
}

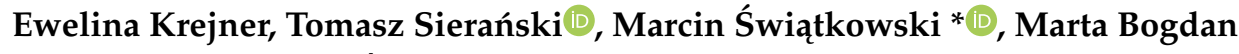 \\ and Rafał Kruszyński ${ }^{\dagger}$ \\ Institute of General and Ecological Chemistry, Lodz University of Technology, Zeromskiego 116, \\ 90-924 Lodz, Poland; ewelina.krejner@edu.p.lodz.pl (E.K.); tomasz.sieranski@p.lodz.pl (T.S.); \\ marta.bogdan@dokt.p.lodz.pl (M.B.) \\ * Correspondence: marcin.swiatkowski@p.lodz.pl; Tel.: +48-42-631-3137 \\ † Rafal Kruszynski deceased in September 2020.
}

Received: 16 October 2020; Accepted: 11 November 2020; Published: 15 November 2020

\begin{abstract}
Two different coordination compounds of copper were synthesized from the same building blocks (1,10-phenanthroline, bromoacetate anions, and copper cations). The synthesis parameters were carefully designed and evaluated to allow the change of the resulting compounds molecular structure, i.e., formation of mononuclear (bromoacetato- $O, O^{\prime}$ ) (bromoacetato- $O$ ) aqua $\left(1,10\right.$-phenanthroline- $\left.N, N^{\prime}\right) \operatorname{copper}(\mathrm{II})$ and dinuclear ( $\mu$-bromido- $\left.1: 2 \kappa^{2}\right)$ bis $\left(\mu\right.$-bromoacetato- $\left.1 \kappa O, 2 \kappa O^{\prime}\right)$ bis(1,10-phenanthroline- $\left.N, N^{\prime}\right)$ dicopper(II) bromoacetate bromoacetic acid solvate. The crystal, molecular and supramolecular structures of the studied compounds were determined and evaluated in Hirshfeld analysis. The UV-Vis-IR absorption and thermal properties were studied and discussed. For the explicit determination of the influence of compounds structure on radiation absorption in UV-Vis range, density functional theory and time-dependent density functional theory calculations were performed.
\end{abstract}

Keywords: copper; bromoacetate; coordination compound; UV-Vis; IR; thermal analysis

\section{Introduction}

The synthesis of coordination compounds with the intended structure is a challenging process [1,2]. Metal centers can adopt diverse coordination numbers (c.n.) and may posses various oxidation states [3-5]. As a specific example, a copper cation can create classical coordination compounds with the c.n. from 2 [6] to 8 [7], and with over a dozen of covalently bonded atoms for copper clusters [8]. For c.n. $=6$, and for six different ligands, 30 different isomers may be created. When the c.n. is higher, the number of possible isomers increases drastically. The copper usually exists in the form of $\mathrm{Cu}^{+}$and $\mathrm{Cu}^{2+}$ cations, but species such as $\mathrm{Cu}^{3+}$ and $\mathrm{Cu}^{4+}$ also exist $[9,10]$. Each can adopt a whole variety of coordination polyhedra [11-14]. Even small changes in the structure of a coordination unit may significantly influence the physicochemical properties of a compound.

The coordination compound formation in a solution depends on many factors such as stoichiometry, concentration, ionic strength, solvent type, and temperature $[15,16]$. Typically, different coordination species exist in an equilibrium in a solution, but not necessarily the dominant one can crystallize. Hence, a proper crystallization environment may allow the production of forms faintly existing or not detectable in the solution (e.g., due to the ions' replacement on the crystal surface during crystal formation).

In this work, the formation of different compounds from the same building blocks was studied. As a ligand, the bromoacetate anion was selected. It possesses multifunctional coordination possibilities, 
and it may be quickly transformed into different species within the specific reaction environment [17]. Bromoacetate anions convert to bromide ions and glycolic acid at elevated temperature or increased concentration/ionic strength. This conversion permits the formation of unique compounds that one cannot synthesize from respective salts [18] and may alter the simple mononuclear coordination moieties to more complex. The additional chelating ligand was used to avoid simple infinite propagation of the coordination via a double bridged carboxylate group. For this purpose, the 1,10-phenanthroline (phen) was selected as its usefulness for breakage (partially or totally) of multinuclear compounds formation was already proven in different systems [19-22]. The applied strategy allowed the synthesis of two new coordination compounds from building blocks used previously to synthesize mononuclear compounds. This work broadens the knowledge of constructing different compounds from the same building units and shows how the alteration of metal centers coordination influences compound properties changes (electronic and thermal). It also highlights difficulties that one may encounter during the analysis of the spectroscopic properties of open-shell systems.

\section{Results and Discussion}

\subsection{Synthesis}

The reaction between copper(II) bromoacetate and 1,10-phenanthroline (phen) leads to the subsequent formation and crystallization of two new coordination compounds, i.e., (bromoacetato-

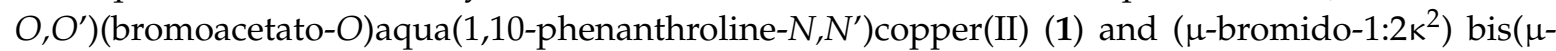
bromoacetato- $\left.1 \kappa \mathrm{K}, 2 \kappa \mathrm{O}^{\prime}\right)$ bis $\left(1,10\right.$-phenanthroline- $\left.N, N^{\prime}\right)$ dicopper(II) bromoacetate bromoacetic acid solvate (2), or to the formation of only one compound (1), depending on the starting concentration of the solution. The larger concentration leads to partial decomposition of the bromoacetate ions due to the larger ionic strength of a solution and subsequently allows the formation of compound 2 in the synthesis S2. An increase in ionic strength allows the decomposition of bromoacetate ions and speeds up this process $[17,18]$. Consequently, $\mathbf{1}$ and $\mathbf{2}$ were formed simultaneously in solution S2B. The bromide ions were detected in the synthesis S2B after stirring. However, they were absent even at the end of crystallization after the synthesis S1B. The earlier formation of the crystals of 2 than the crystals of 1 originates from different solubility of both compounds in water (approximate values 2.1 and $8.0 \mathrm{~g} / 100 \mathrm{~cm}^{3}$ of used solvent mixture, respectively). After forming and separating the crystals of $\mathbf{2}$, the bromide ions were absent in the solution (the formed bromide ions were utilized in the formation of $\mathbf{2}$, and further decomposition of bromoacetate ions did not occur due to the lowering of solution ionic strength after crystallization of 2). The phase purity of each crystal fraction was confirmed by comparing the high-resolution IR spectra registered for the crystal with the determined structure and for the bulk sample (the differences allowing unambiguous assignment of phases are described in paragraph 3.4 and collected in Table S4). The studied compounds possess 1:1 M:L stoichiometry, but they were formed only from 2:1 M:L stoichiometry of the substrates. Application during synthesis 1:1 M:L stoichiometry led to the formation of literature-known $\left[\mathrm{CuBr}(\mathrm{phen})_{2}\right]^{+} \bullet \mathrm{Br}^{-}$hydrate [23]. This is caused by large stability constants of $\mathrm{Cu}$-phen systems $\left(\log \beta_{\mathrm{Cu} \text { (phen) }}=9.25, \log \beta_{\mathrm{Cu}(\mathrm{phen}) 2}=16.0\right.$, $\log \beta_{\mathrm{Cu}(\mathrm{phen}) 3}=21.35$ ) [24], and necessity of ligand deficiency in a reaction system for the formation of the compound with 1:1 M:L stoichiometry, i.e., shifting of equilibria toward the formation of $\mathrm{Cu}$ (phen) ${ }^{2+}$ species (instead of $\mathrm{Cu}$ (phen $)_{2}{ }^{2+}$ and $\mathrm{Cu}$ (phen $)_{3}{ }^{2+}$ species) by decreasing the concentration of phen. In the literature, the description of the synthesis of $\left[\mathrm{Cu}\left(\mathrm{BrCH}_{2} \mathrm{COO}\right)_{2}(\mathrm{phen})\right]$ compound in hydrothermal conditions can be found [25]. Nevertheless, repeating this synthesis (for details see Supplementary Materials) leads to the formation of [CuBr(phen $\left.)_{2}\right]^{+} \bullet \mathrm{Br}^{-}$hydrate [23] instead of $\left[\mathrm{Cu}\left(\mathrm{BrCH}_{2} \mathrm{COO}\right)_{2}\right.$ (phen)]. It might originate from different conditions in the original and repeated syntheses (current work), as in the original paper [25] experimental details (e.g., temperature and time of reaction, a volume of used vessel, reached pressure within the vessel) were not provided. 


\subsection{Structural Analysis}

Compound $\mathbf{1}$ is a molecular system possessing one coordination moiety located in an asymmetric unit and no ligands in an outer coordination sphere (Figure 1). Compound $\mathbf{2}$ is distinctly more complex. Its coordination moiety is dinuclear and occupies two asymmetric units (Figure 1). All except one atom of 2 lie in general positions. The presence of the twofold rotation axis (special position e of $C 2 / c$ space group with multiplicity 4) going through Br2 ion causes the dinuclear complex cation to be composed of two symmetry dependent bridging bromoacetate ions, two chelating phen molecules, two copper cations and one bridging bromide ion. The observed dinuclear triple-bridged core is unique among copper-phen coordination compounds (among 3415 structurally determined copper-phen compound [26] none possesses bridges formed by two carboxylate ions and one halogen ion). The outer coordination sphere of $\mathbf{2}$ contains one bromoacetate ion balancing the complex cation charge and one neutral bromoacetic acid molecule (each existing in different asymmetric units). The inversion center (special position d with multiplicity 4) between neighboring bromoacetate anion and bromoacetic acid molecules leads to disorder of the carboxylic hydrogen atom equally over two positions and the formal presence of half of the anion as well as half of the acid in one asymmetric unit.

The copper atom of $\mathbf{1}$ is six-coordinated and adopts a distorted tetragonal bipyramid geometry with $\mathrm{O} 1$ and $\mathrm{N} 2$ atoms located at the polyhedron apexes (Figure 2a) [27]. The Cu1-O4 coordination bond (Table 1) is distinctly longer than other ones, but it still falls in the range of the sum of van der Waals radii (equal 2.9 $\AA$ according to Bondi or 3.2 according to Zefirov) $[28,29]$. The bond valence analysis [30-33] shows that BVS (the sum of bond valences, Table 1 ) in $\mathbf{1}$ is equal to 1.894 for presumed c.n. $=5$ and 1.974 for c.n. $=6$. It proves the conclusion about the six-coordinated copper atom of $\mathbf{1}$ based on the bond lengths. The central atoms of 2 are five-coordinated and exist in a transitional coordination geometry between square pyramidal (Figure 2b) and trigonal-bipyramidal (Figure 2c) [34]. The observed distortion from an ideal polyhedron geometry observed in both compounds is caused by the rigid and flat structure of the chelating phen molecules and the restraints provided by the chelating (in 1) and bridging (in 2) carboxylate anions. The large structural strains occurring around central atoms disallow occupation of ideal positions by the coordinating atoms of the ligands. The chelating bromoacetate anion of $\mathbf{1}$ asymmetrically bonds to the copper cation, and the bond length difference is $0.77 \AA$ (Table 1). Oppositely, in 2 the bridging bromoacetate anion shows symmetricity of the formed coordination bonds. 
1

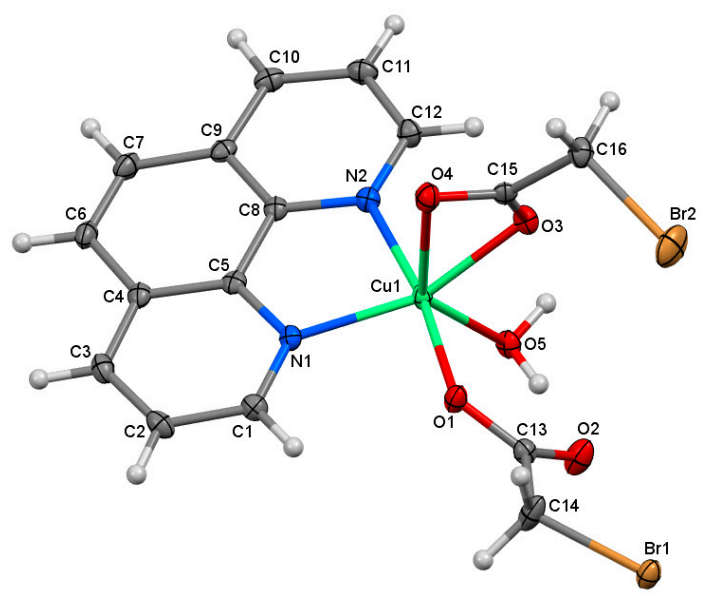

2
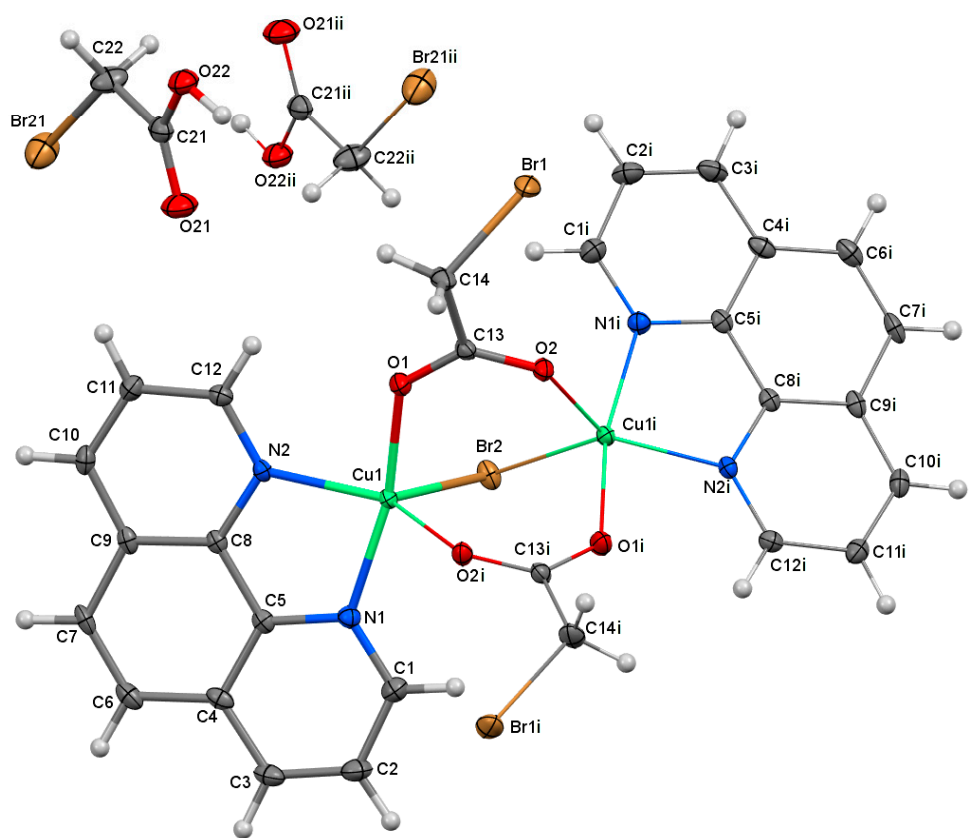

Figure 1. Solid state structures of the studied compounds, with atom numbering scheme, plotted with $50 \%$ probability of displacement ellipsoids of non-hydrogen atoms. Hydrogen atoms are plotted as spheres of arbitrary radii. The symmetry generated atoms are indicated by $i$ and ii letters [symmetry code: (i) $-x+1, y,-z+1.5$; (ii) $-x+0.5,-y+0.5,-z+1]$. The occupancy of hydrogen atom bonded to $\mathrm{O} 22$ is set on 0.5 (see Section 3.2).

(a)

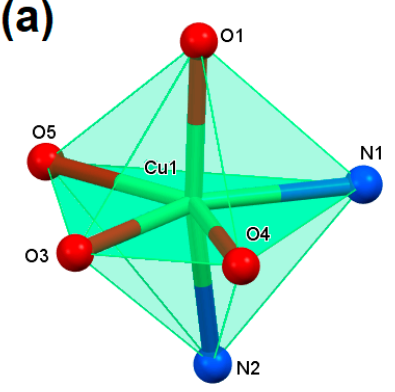

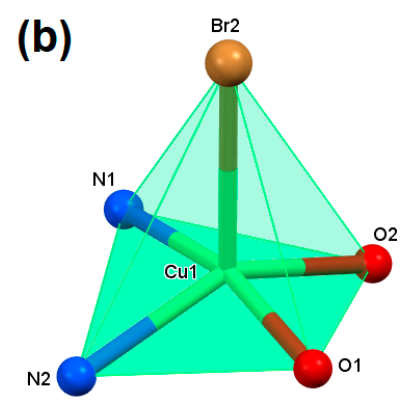

Figure 2. Coordination polyhedra of the compound $\mathbf{1}(\mathbf{a})$ and $\mathbf{2}(\mathbf{b}-\mathbf{c})$. For a description, see the text. 
Table 1. Selected structural data of the studied compounds.

\begin{tabular}{|c|c|c|c|c|c|c|}
\hline $\mathrm{i}-\mathrm{j}$ & $\begin{array}{l}\mathrm{d}_{\mathrm{ij}} \\
(\stackrel{\mathrm{A}}{\mathrm{i}})\end{array}$ & $\begin{array}{c}v_{\mathrm{ij}} \\
\text { (v.u.) }\end{array}$ & $\mathrm{i}-\mathrm{j}-\mathrm{k}$ & $\alpha_{\mathrm{ijk}}^{\left.{ }^{\circ}\right)}$ & $\mathrm{i}-\mathrm{j}-\mathrm{k}$ & $\begin{array}{c}\alpha_{\mathrm{ijk}} \\
\left(^{\circ}\right)\end{array}$ \\
\hline \multicolumn{7}{|c|}{ compound 1} \\
\hline $\mathrm{Cu} 1-\mathrm{N} 1$ & $2.0255(17)$ & $0.432 * / 0.421$ & $\mathrm{~N} 1-\mathrm{Cu} 1-\mathrm{N} 2$ & $81.77(7)$ & $\mathrm{N} 2-\mathrm{Cu} 1-\mathrm{O} 5$ & $90.27(6)$ \\
\hline $\mathrm{Cu} 1-\mathrm{N} 2$ & 2.0112(17) & $0.449 * / 0.437$ & $\mathrm{~N} 1-\mathrm{Cu} 1-\mathrm{O} 1$ & $89.34(6)$ & $\mathrm{O} 1-\mathrm{Cu} 1-\mathrm{O} 3$ & $91.68(6)$ \\
\hline $\mathrm{Cu} 1-\mathrm{O} 1$ & $1.9428(14)$ & $\begin{array}{c}0.461 * / 0.456 \\
+\end{array}$ & $\mathrm{N} 1-\mathrm{Cu} 1-\mathrm{O} 3$ & $150.23(6)$ & $\mathrm{O} 1-\mathrm{Cu} 1-\mathrm{O} 4$ & $83.82(6)$ \\
\hline $\mathrm{Cu} 1-\mathrm{O} 3$ & $2.0263(14)$ & $\begin{array}{c}0.368 * / 0.364 \\
t\end{array}$ & $\mathrm{~N} 1-\mathrm{Cu} 1-\mathrm{O} 4$ & $98.38(6)$ & $\mathrm{O} 1-\mathrm{Cu} 1-\mathrm{O} 5$ & $93.17(6)$ \\
\hline $\mathrm{Cu} 1-\mathrm{O} 4$ & 2.7977(14) & $0.046^{*}$ & $\mathrm{~N} 1-\mathrm{Cu} 1-\mathrm{O} 5$ & $118.85(6)$ & $\mathrm{O} 3-\mathrm{Cu} 1-\mathrm{O} 4$ & $52.25(6)$ \\
\hline \multirow[t]{2}{*}{$\mathrm{Cu} 1-\mathrm{O} 5$} & $2.2175(14)$ & $\begin{array}{c}0.219 * / 0.217 \\
+\end{array}$ & $\mathrm{N} 2-\mathrm{Cu} 1-\mathrm{O} 1$ & $171.06(7)$ & $\mathrm{O} 3-\mathrm{Cu} 1-\mathrm{O} 5$ & $90.81(6)$ \\
\hline & & & $\begin{array}{l}\mathrm{N} 2-\mathrm{Cu} 1-\mathrm{O} 3 \\
\mathrm{~N} 2-\mathrm{Cu} 1-\mathrm{O} 4\end{array}$ & $\begin{array}{l}96.52(6) \\
98.40(6)\end{array}$ & $\mathrm{O} 4-\mathrm{Cu} 1-\mathrm{O} 5$ & $142.65(7)$ \\
\hline \multicolumn{7}{|c|}{ compound 2} \\
\hline $\mathrm{Cu} 1-\mathrm{N} 1$ & $2.001(2)$ & 0.449 & $\mathrm{~N} 1-\mathrm{Cu} 1-\mathrm{N} 2$ & $81.81(9)$ & $\mathrm{N} 2-\mathrm{C}_{\mathrm{i}} \mathrm{1} 1-\mathrm{O} 2$ & $139.74(8)$ \\
\hline $\mathrm{Cu} 1-\mathrm{N} 2$ & $2.033(2)$ & 0.412 & $\mathrm{~N} 1-\mathrm{Cu} 1-\mathrm{O} 1$ & $166.12(8)$ & $\mathrm{N} 2-\mathrm{Cu} 1-\mathrm{Br} 2$ & $114.14(6)$ \\
\hline $\mathrm{Cu} 1-\mathrm{O} 1$ & $1.9378(19)$ & 0.462 & $\mathrm{~N} 1-\underset{\mathrm{i}}{\mathrm{C}} \mathrm{1} 1-\mathrm{O} 2$ & $88.52(8)$ & $\mathrm{O} 1-\underset{\mathrm{i}}{\mathrm{C}} \mathrm{1} 1-\mathrm{O} 2$ & $93.35(8)$ \\
\hline $\mathrm{Cu} 1-\mathrm{O} 2^{\mathrm{i}}$ & $1.9964(18)$ & 0.394 & $\mathrm{~N} 1-\mathrm{Cu} 1-\mathrm{Br} 2$ & $95.42(6)$ & $\mathrm{O} 1-\mathrm{Cu} 1-\mathrm{Br} 2$ & $97.31(6)$ \\
\hline $\mathrm{Cu} 1-\mathrm{Br} 2$ & $2.6198(4)$ & 0.270 & $\mathrm{~N} 2-\mathrm{Cu} 1-\mathrm{O} 1$ & $87.95(8)$ & $\stackrel{\mathrm{O} 2}{\mathrm{i}-\mathrm{Cu} 1-\mathrm{Br} 2}$ & $105.60(5)$ \\
\hline
\end{tabular}

Bond valences $\left(v_{\mathrm{ij}}\right)$ calculated with use of parameters for ${ }^{*}$ six-coordinated and + five-coordinated copper(II). Symmetry transformations used to generate equivalent atoms: (i) $-\mathrm{x}+1, \mathrm{y},-\mathrm{z}+1.5$.

The supramolecular structures of the studied compounds are stabilized by $\mathrm{O}-\mathrm{H} \bullet \bullet \bullet \mathrm{O}, \mathrm{C}-\mathrm{H} \bullet \bullet \bullet \mathrm{O}$ and $\mathrm{C}-\mathrm{H} \bullet \bullet \bullet \mathrm{Br}$ hydrogen bonds (Table S1). In 1, the unitary graph set of classical hydrogen bonds comprises $\mathrm{S}(6)$ and $\mathrm{R}_{2}{ }^{2}(8)$ motif. In 2 , it contains only one finite $\mathrm{D}(2)$ motif. Both $\mathrm{S}$ and $\mathrm{R}$ motifs engage water molecules as hydrogen bond donors and carboxylate ions as acceptors. The $S$ motif is formed by $\mathrm{O}-\mathrm{H} \bullet \bullet \bullet \mathrm{O}$ interaction between water molecules and carboxylate ions coordinated to the same copper cation while $\mathrm{R}$ motif is formed by $\mathrm{O}-\mathrm{H} \bullet \bullet \bullet \mathrm{O}$ hydrogen bonds between the neighboring coordination units, linking them into a supramolecular dimer. In 2, the present $\mathrm{D}(2)$ motif is formed only by the interaction between species present in the outer coordination sphere-a characteristic anti-anti type arrangement for carboxylate•••carboxylic acid interactions [35]. The molecules of $\mathbf{1}$ are stacked to the piles extending along [100] crystallographic axis via $\pi \bullet \bullet \bullet \pi$ interactions between aromatic rings of phen (Table S2, Figure S6). Each six-membered ring interacts with a total of four neighboring rings. One of these rings belongs to one of the adjacent phen molecules in the pyridine case, and three others belong to second nearby phen molecules. Each benzene ring always interacts with two rings of each neighboring phen molecule. The molecules of 2 are stacked to the layers expanding along (1 000$)$ crystallographic plane due to the presence of two phen ligands in the coordination unit (the phen molecules themselves are assembled to piles propagating along [010] crystallographic axis via $\pi \bullet \bullet \pi$ interactions between aromatic rings of phen; Figure S6). In 2, only one six-membered ring (i.e., the pyridine ring containing $\mathrm{N} 1$ atom) interacts with four neighboring rings, and two other rings interact with six adjacent rings of two symmetry-related phen molecules. In both compounds, the subsequent phen molecules are packed alternatively in the crystal net (succeeding phen molecules are reversed along bond linking pyridine rings). The intermolecular interactions were also analysed based on Hirshfeld surfaces and fingerprints (see Supplementary Materials for details, Figures S1-S5 and S7-S8). 


\subsection{UV-Vis Spectra Analysis}

The calculated spectrum of $\mathbf{1}$ is similar to the experimental one. Noticeable differences exist in the case of 2 (Figure 3, Figure S14, Table 2). TD-DFT works better for closed-shell systems [36]. Both $\mathbf{1}$ and $\mathbf{2}$ contain open-shell centers. However, the case of $\mathbf{2}$ is more complicated. The centers in 2 can produce a singlet-state molecule with unpaired electrons of two $\mathrm{Cu}^{2+}$ possessing opposite spins, or they can form a triplet state molecule with unpaired electrons of two $\mathrm{Cu}^{2+}$ possessing the same spins. Therefore, the spectrum of $\mathbf{2}$ was more challenging to reproduce. All applied approaches (Figure S14) led to different spectra. For UB3LYP approach, the number of the observed maxima and their mutual arrangement are accurately reproduced and reasonably reflect the experimental ones. Yet, this approach alone does not give a system for which its electronic state's energy is the lowest. Only the optimization of a wave function led to a stable wave function and gave a state with the lowest energy (an antiferromagnetic singlet state, Table S3).
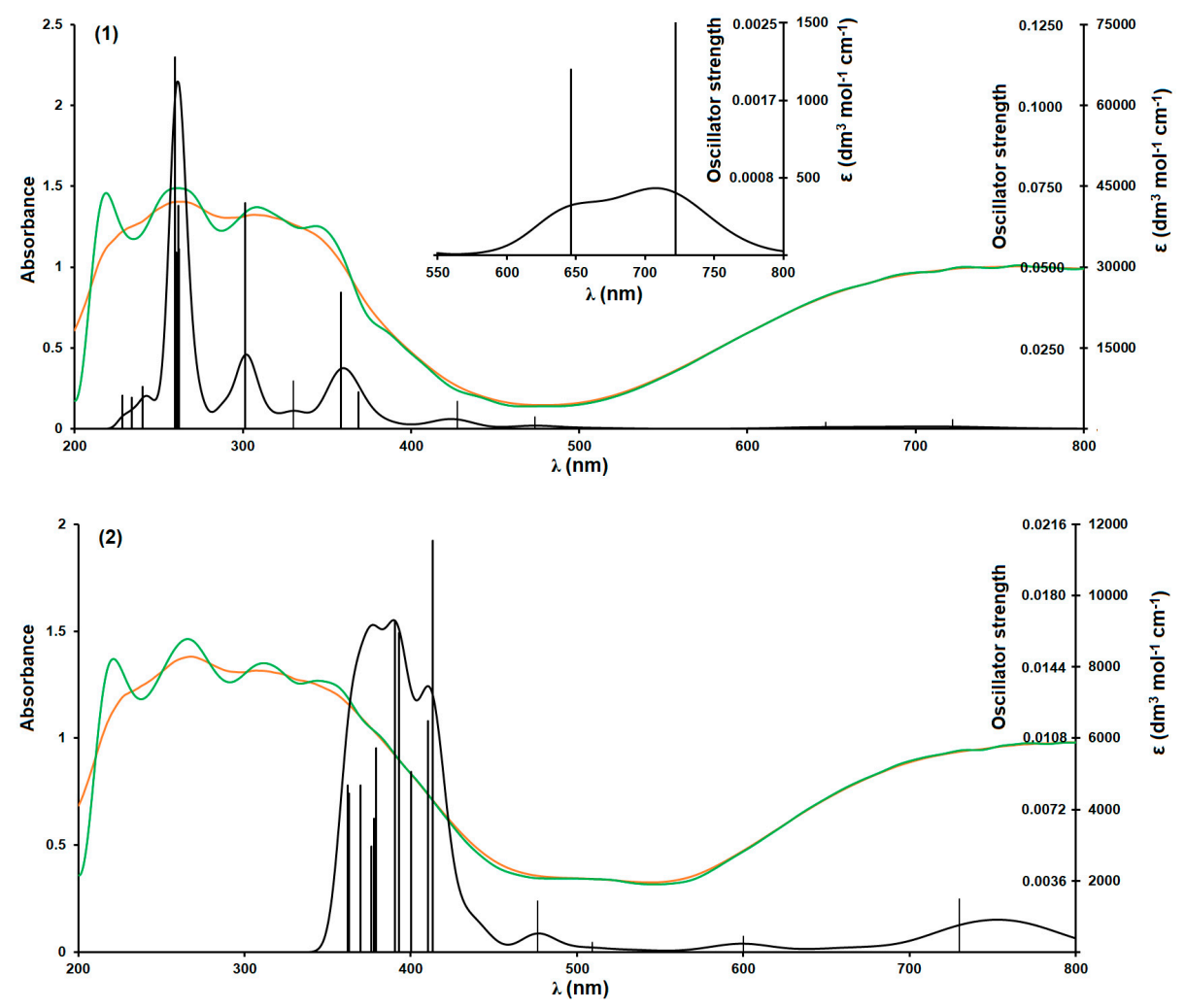

Figure 3. Experimental (orange-registered, green-deconvoluted) and calculated (black) UV-Vis spectra of the studied compounds ( $\mathbf{1}$ and $\mathbf{2}$ ). The most important oscillator strengths are shown as vertical black lines. 
Table 2. The most important electronic transitions. H letter indicate HOMO, L-LUMO, $\alpha$ - $\alpha$ orbitals, $\beta-\beta$ orbitals, and $\pm($ number) represents subsequent orbitals above HOMO and LUMO, respectively.

\begin{tabular}{|c|c|c|c|c|c|c|c|c|}
\hline \multicolumn{2}{|c|}{ Theoretical $\lambda(\mathrm{nm})$} & \multirow{2}{*}{$\mathrm{E}(\mathrm{eV})$} & \multirow{2}{*}{$f$} & \multirow{2}{*}{$\begin{array}{l}\text { The Most Important Orbitals } \\
\text { Involved in Electronic Transitions }\end{array}$} & \multirow{2}{*}{ Character of Transition } & \multicolumn{3}{|c|}{$\begin{array}{l}\text { Experimental } \lambda(\mathrm{nm}) \\
\quad(\text { Solid State) }\end{array}$} \\
\hline 1 & 2 & & & & & 1 & 2 & phen \\
\hline 228.31 & & 5.4305 & 0.0104 & $\begin{array}{c}\alpha \mathrm{H}-5 \rightarrow \alpha \mathrm{L}+3 \\
\beta \mathrm{H}-15 \rightarrow \beta \mathrm{L}\end{array}$ & $\begin{array}{c}\mathrm{d}(\mathrm{Cu}) / \mathrm{n}(\mathrm{BrAcO}) / \sigma(\mathrm{BrAcO}) \rightarrow \pi^{*}(\text { phen }) \\
\mathrm{d}(\mathrm{Cu}) / \mathrm{n}(\mathrm{BrAcO}) / \sigma(\mathrm{BrAcO}) / \pi(\text { phen }) \rightarrow \mathrm{d}(\mathrm{Cu}) / \pi^{*}(\text { phen })\end{array}$ & \multirow{6}{*}{218.99} & \multirow{6}{*}{220.84} & \multirow{6}{*}{214} \\
\hline 233.98 & & 5.2989 & 0.0096 & $\alpha \mathrm{H}-9 \rightarrow \alpha \mathrm{L}+2$ & $\mathrm{~d}(\mathrm{Cu}) / \mathrm{n}(\mathrm{BrAcO}) / \pi($ phen $) \rightarrow \pi^{*}($ phen $)$ & & & \\
\hline \multirow[t]{4}{*}{240.36} & & 5.1583 & 0.0130 & $\beta \mathrm{H}-8 \rightarrow \beta \mathrm{L}+3$ & $\mathrm{~d}(\mathrm{Cu}) / \mathrm{n}(\mathrm{BrAcO}) / \pi($ phen $) \rightarrow \pi^{*}($ phen $)$ & & & \\
\hline & 362.12 & 3.4238 & 0.0085 & $\begin{array}{c}\beta \mathrm{H}-17 \rightarrow \beta \mathrm{L} \\
\beta \mathrm{H}-8 \rightarrow \beta \mathrm{L}+3\end{array}$ & $\begin{array}{c}\pi(\text { phen }) \rightarrow \mathrm{d}(\mathrm{Cu}) / \sigma^{*}(\text { phen }) / \sigma^{*}(\mathrm{BrAcO}) \\
\mathrm{d}(\mathrm{Cu}) / \mathrm{n}(\mathrm{Br}) / \mathrm{n}(\mathrm{BrAcO}) \rightarrow \pi^{*}(\text { phen })\end{array}$ & & & \\
\hline & 363.01 & 3.4154 & 0.0081 & $\begin{array}{c}\beta \mathrm{H}-17 \rightarrow \beta \mathrm{L} \\
\beta \mathrm{H}-8 \rightarrow \beta \mathrm{L}+3\end{array}$ & $\begin{array}{l}\pi(\mathrm{phen}) \rightarrow \mathrm{d}(\mathrm{Cu}) / \sigma^{*}(\mathrm{phen}) / \sigma^{*}(\mathrm{BrAcO}) \\
\mathrm{d}(\mathrm{Cu}) / \mathrm{n}(\mathrm{Br}) / \mathrm{n}(\mathrm{BrAcO}) \rightarrow \pi^{*}(\text { phen })\end{array}$ & & & \\
\hline & 369.66 & 3.3540 & 0.0085 & $\beta \mathrm{H}-13 \rightarrow \beta \mathrm{L}$ & $\mathrm{d}(\mathrm{Cu}) / \mathrm{n}(\mathrm{Br}) / \mathrm{n}(\mathrm{BrAcO}) / \pi($ phen $) \rightarrow \mathrm{d}(\mathrm{Cu}) / \sigma^{*}($ phen $) / \sigma^{*}(\mathrm{BrAcO})$ & & & \\
\hline 259.56 & & 4.7767 & 0.1149 & $\beta \mathrm{H}-13 \rightarrow \beta \mathrm{L}$ & $\mathrm{d}(\mathrm{Cu}) / \mathrm{n}(\mathrm{BrAcO}) \rightarrow \mathrm{d}(\mathrm{Cu}) / \pi^{*}($ phen $)$ & \multirow{8}{*}{261.35} & \multirow{8}{*}{265.62} & \multirow{8}{*}{253} \\
\hline 260.46 & & 4.7602 & 0.0545 & $\alpha \mathrm{H}-1 \rightarrow \alpha \mathrm{L}+3$ & $\mathrm{~d}(\mathrm{Cu}) / \mathrm{n}(\mathrm{BrAcO}) / \sigma(\mathrm{BrAcO}) \rightarrow \pi^{*}($ phen $)$ & & & \\
\hline 261.78 & & 4.7362 & 0.0689 & $\beta \mathrm{H}-5 \rightarrow \beta \mathrm{L}+3$ & $\mathrm{~d}(\mathrm{Cu}) / \mathrm{n}(\mathrm{BrAcO}) / \sigma(\mathrm{BrAcO}) \rightarrow \pi^{*}($ phen $)$ & & & \\
\hline 262.05 & & 4.7314 & 0.0555 & $\beta \mathrm{H}-5 \rightarrow \beta \mathrm{L}+3$ & $\mathrm{~d}(\mathrm{Cu}) / \mathrm{n}(\mathrm{BrAcO}) / \sigma(\mathrm{BrAcO}) \rightarrow \pi^{*}($ phen $)$ & & & \\
\hline \multirow[t]{4}{*}{262.65} & & 4.7206 & 0.0572 & $\alpha \mathrm{H}-5 \rightarrow \alpha \mathrm{L}+2$ & $\mathrm{~d}(\mathrm{Cu}) / \mathrm{n}(\mathrm{BrAcO}) / \sigma(\mathrm{BrAcO}) \rightarrow \pi^{*}($ phen $)$ & & & \\
\hline & 376.25 & 3.2953 & 0.0054 & $\beta \mathrm{H}-6 \rightarrow \beta \mathrm{L}+3$ & $\mathrm{~d}(\mathrm{Cu}) / \mathrm{n}(\mathrm{Br}) / \mathrm{n}(\mathrm{BrAcO}) \rightarrow \pi^{*}($ phen $)$ & & & \\
\hline & 378.12 & 3.2789 & 0.0068 & $\alpha \mathrm{H}-13 \rightarrow \alpha \mathrm{L}$ & $\mathrm{d}(\mathrm{Cu}) / \mathrm{n}(\mathrm{Br}) / \mathrm{n}(\mathrm{BrAcO}) / \pi(\mathrm{phen}) \rightarrow \mathrm{d}(\mathrm{Cu}) / \sigma^{*}($ phen $) / \sigma^{*}(\mathrm{BrAcO})$ & & & \\
\hline & 379.21 & 3.2696 & 0.0104 & $\beta \mathrm{H}-6 \rightarrow \beta \mathrm{L}+3$ & $\mathrm{~d}(\mathrm{Cu}) / \mathrm{n}(\mathrm{Br}) / \mathrm{n}(\mathrm{BrAcO}) \rightarrow \pi^{*}($ phen $)$ & & & \\
\hline 301.36 & & 4.1142 & 0.0697 & $\beta \mathrm{H}-9 \rightarrow \beta \mathrm{L}+2$ & $\mathrm{~d}(\mathrm{Cu}) / \mathrm{n}\left(\mathrm{H}_{2} \mathrm{O}\right) \mathrm{n}(\mathrm{BrAcO}) \sigma(\mathrm{BrAcO}) / \pi($ phen $) \rightarrow \mathrm{d}(\mathrm{Cu}) / \sigma^{*}($ phen $) / \sigma^{*}(\mathrm{BrAcO})$ & \multirow{5}{*}{308.46} & \multirow{5}{*}{310.77} & \multirow{5}{*}{294} \\
\hline \multirow[t]{4}{*}{330.05} & & 3.7565 & 0.0147 & $\begin{array}{c}\beta \mathrm{H}-7 \rightarrow \beta \mathrm{L}+2 \\
\beta \mathrm{H}-8 \rightarrow \beta \mathrm{L}\end{array}$ & $\begin{array}{c}\mathrm{d}(\mathrm{Cu}) / \mathrm{n}\left(\mathrm{H}_{2} \mathrm{O}\right) \mathrm{n}(\mathrm{BrAcO}) \rightarrow \mathrm{d}(\mathrm{Cu}) / \sigma^{*}(\text { phen }) / \sigma^{*}(\mathrm{BrAcO}) \\
\mathrm{d}(\mathrm{Cu}) / \mathrm{n}(\mathrm{BrAcO}) / \pi(\text { phen }) \rightarrow \mathrm{d}(\mathrm{Cu}) / \pi^{*}(\text { phen })\end{array}$ & & & \\
\hline & 390.59 & 3.1743 & 0.0169 & $\begin{array}{l}\alpha \mathrm{H}-11 \rightarrow \alpha \mathrm{L} \\
\beta \mathrm{H}-15 \rightarrow \beta \mathrm{L}\end{array}$ & $\begin{array}{c}\mathrm{n}(\mathrm{Br}) / \mathrm{n}(\mathrm{BrAcO}) / \sigma(\mathrm{BrAcO}) \rightarrow \mathrm{d}(\mathrm{Cu}) / \sigma^{*}(\mathrm{phen}) / \sigma^{*}(\mathrm{BrAcO}) \\
\mathrm{d}(\mathrm{Cu}) /(\mathrm{Br}) / \mathrm{n}(\mathrm{BrAcO}) / \sigma(\mathrm{phen}) \rightarrow \mathrm{d}(\mathrm{Cu}) / \sigma^{*}(\text { phen }) / \sigma^{*}(\mathrm{BrAcO})\end{array}$ & & & \\
\hline & 392.79 & 3.1565 & 0.0163 & $\alpha \mathrm{H}-11 \rightarrow \alpha \mathrm{L}$ & $\mathrm{n}(\mathrm{Br}) / \mathrm{n}(\mathrm{BrAcO}) / \sigma(\mathrm{BrAcO}) \rightarrow \mathrm{d}(\mathrm{Cu}) / \sigma^{*}($ phen $) / \sigma^{*}(\mathrm{BrAcO})$ & & & \\
\hline & 400.16 & 3.0984 & 0.0092 & $\alpha \mathrm{H}-9 \rightarrow \alpha \mathrm{L}$ & $\mathrm{n}(\mathrm{Br}) / \mathrm{n}(\mathrm{BrAcO}) / \sigma(\mathrm{BrAcO}) \rightarrow \mathrm{d}(\mathrm{Cu}) / \sigma^{*}(\mathrm{phen}) / \sigma^{*}(\mathrm{BrAcO})$ & & & \\
\hline
\end{tabular}


Table 2. Cont

\begin{tabular}{|c|c|c|c|c|c|c|c|c|}
\hline \multicolumn{2}{|c|}{ Theoretical $\lambda(\mathrm{nm})$} & \multirow[t]{2}{*}{$\mathrm{E}(\mathrm{eV})$} & \multirow{2}{*}{$f$} & \multirow{2}{*}{$\begin{array}{l}\text { The Most Important Orbitals } \\
\text { Involved in Electronic Transitions }\end{array}$} & \multirow{2}{*}{ Character of Transition } & \multicolumn{3}{|c|}{$\begin{array}{l}\text { Experimental } \lambda(\mathrm{nm}) \\
\quad(\text { Solid State) }\end{array}$} \\
\hline 1 & 2 & & & & & 1 & 2 & phen \\
\hline 358.41 & & 3.4593 & 0.0421 & $\beta \mathrm{H}-4 \rightarrow \beta \mathrm{L}+2$ & $\mathrm{~d}(\mathrm{Cu}) / \mathrm{n}\left(\mathrm{H}_{2} \mathrm{O}\right) / \mathrm{n}(\mathrm{BrAcO}) / \sigma(\mathrm{BrAcO}) \rightarrow \mathrm{d}(\mathrm{Cu}) / \sigma^{*}(\mathrm{phen}) / \sigma^{*}(\mathrm{BrAcO})$ & \multirow{4}{*}{$\begin{array}{l}344.61 \\
384.41\end{array}$} & \multirow{4}{*}{344.77} & \multirow{4}{*}{$\begin{array}{l}324 \\
335\end{array}$} \\
\hline \multirow[t]{3}{*}{368.93} & & 3.3606 & 0.0114 & $\begin{array}{l}\beta \mathrm{H}-5 \rightarrow \beta \mathrm{L} \\
\beta \mathrm{H}-4 \rightarrow \beta \mathrm{L}\end{array}$ & $\begin{array}{c}\mathrm{d}(\mathrm{Cu}) / \mathrm{n}(\mathrm{BrAcO}) / \sigma(\mathrm{BrAcO}) \rightarrow \mathrm{d}(\mathrm{Cu}) / \pi^{*}(\text { phen }) \\
\mathrm{d}(\mathrm{Cu}) / \mathrm{n}\left(\mathrm{H}_{2} \mathrm{O}\right) / \mathrm{n}(\mathrm{BrAcO}) / \sigma(\mathrm{BrAcO}) \rightarrow \mathrm{d}(\mathrm{Cu}) / \pi^{*}(\text { phen })\end{array}$ & & & \\
\hline & 410.44 & 3.0207 & 0.0118 & $\begin{array}{l}\beta \mathrm{H}-7 \rightarrow \beta \mathrm{L}+1 \\
\beta \mathrm{H}-8 \rightarrow \beta \mathrm{L}+1\end{array}$ & $\begin{array}{l}\mathrm{d}(\mathrm{Cu}) / \mathrm{n}(\mathrm{Br}) / \mathrm{n}(\mathrm{BrAcO}) \rightarrow \pi^{*}(\text { phen }) \\
\mathrm{d}(\mathrm{Cu}) / \mathrm{n}(\mathrm{Br}) / \mathrm{n}(\mathrm{BrAcO}) \rightarrow \pi^{*}(\text { phen })\end{array}$ & & & \\
\hline & 413.33 & 2.9997 & 0.0210 & $\begin{array}{l}\alpha \mathrm{H}-8 \rightarrow \alpha \mathrm{L}+1 \\
\beta \mathrm{H}-7 \rightarrow \beta \mathrm{L}+1\end{array}$ & $\begin{array}{l}\mathrm{d}(\mathrm{Cu}) / \mathrm{n}(\mathrm{Br}) / \mathrm{n}(\mathrm{BrAcO}) \rightarrow \pi^{*}(\text { phen }) \\
\mathrm{d}(\mathrm{Cu}) / \mathrm{n}(\mathrm{Br}) / \mathrm{n}(\mathrm{BrAcO}) \rightarrow \pi^{*}(\text { phen })\end{array}$ & & & \\
\hline \multirow[t]{2}{*}{427.35} & & & 0.0085 & $\beta \mathrm{H}-2 \rightarrow \beta \mathrm{L}+2$ & $\mathrm{~d}(\mathrm{Cu}) / \mathrm{n}\left(\mathrm{H}_{2} \mathrm{O}\right) \mathrm{n}(\mathrm{BrAcO}) \sigma(\mathrm{BrAcO}) \rightarrow \mathrm{d}(\mathrm{Cu}) / \sigma^{*}(\mathrm{phen}) / \sigma^{*}(\mathrm{BrAcO})$ & 439.55 & & \\
\hline & 476.36 & 2.6027 & 0.0026 & $\begin{array}{l}\alpha \mathrm{H}-6 \rightarrow \alpha \mathrm{L} \\
\alpha \mathrm{H}-7 \rightarrow \alpha \mathrm{L}\end{array}$ & $\begin{array}{l}\mathrm{d}(\mathrm{Cu}) / \mathrm{n}(\mathrm{Br}) / \mathrm{n}(\mathrm{BrAcO}) \rightarrow \mathrm{d}(\mathrm{Cu}) / \sigma^{*}(\text { phen }) / \sigma^{*}(\mathrm{BrAcO}) \\
\mathrm{d}(\mathrm{Cu}) / \mathrm{n}(\mathrm{Br}) / \mathrm{n}(\mathrm{BrAcO}) \rightarrow \mathrm{d}(\mathrm{Cu}) / \sigma^{*}(\text { phen }) / \sigma^{*}(\mathrm{BrAcO})\end{array}$ & & 516.59 & \\
\hline 473.81 & & 2.6167 & 0.0037 & $\alpha \mathrm{H}-1 \rightarrow \alpha \mathrm{L}$ & $\mathrm{d}(\mathrm{Cu}) / \mathrm{n}(\mathrm{BrAcO}) / \sigma(\mathrm{BrAcO}) \rightarrow \mathrm{d}(\mathrm{Cu}) / \mathrm{n}(\mathrm{BrAcO}) / \sigma^{*}(\mathrm{BrAcO}) / \sigma^{*}(\mathrm{phen})$ & \multirow{7}{*}{$\begin{array}{l}726.94 \\
759.80\end{array}$} & \multirow{7}{*}{$\begin{array}{l}728.98 \\
769.21\end{array}$} & \\
\hline 646.65 & & 1.9173 & 0.0020 & $\begin{array}{l}\beta \mathrm{H}-14 \rightarrow \beta \mathrm{L}+2 \\
\beta \mathrm{H}-13 \rightarrow \beta \mathrm{L}+2\end{array}$ & $\begin{array}{c}\mathrm{d}(\mathrm{Cu}) / \mathrm{n}\left(\mathrm{H}_{2} \mathrm{O}\right) / \sigma(\mathrm{BrAcO}) / \pi(\mathrm{phen}) \rightarrow \mathrm{d}(\mathrm{Cu}) / \sigma^{*}(\text { phen }) / \sigma^{*}(\mathrm{BrAcO}) \\
\mathrm{d}(\mathrm{Cu}) / \mathrm{n}(\mathrm{BrAcO}) \rightarrow \mathrm{d}(\mathrm{Cu}) / \sigma^{*}(\mathrm{phen}) / \sigma^{*}(\mathrm{BrAcO})\end{array}$ & & & \\
\hline 712.92 & & 1.7391 & 0.0028 & $\beta \mathrm{H}-13 \rightarrow \beta \mathrm{L}+2$ & $\mathrm{~d}(\mathrm{Cu}) / \mathrm{n}(\mathrm{BrAcO}) \rightarrow \mathrm{d}(\mathrm{Cu}) / \sigma^{*}($ phen $) / \sigma^{*}(\mathrm{BrAcO})$ & & & \\
\hline 949.37 & & 1.3060 & 0.0028 & $\beta \mathrm{H} \rightarrow \beta \mathrm{L}+2$ & $\mathrm{~d}(\mathrm{Cu}) / \mathrm{n}(\mathrm{BrAcO}) \rightarrow \mathrm{d}(\mathrm{Cu}) / \sigma^{*}($ phen $) / \sigma^{*}(\mathrm{BrAcO})$ & & & \\
\hline & 509.01 & 2.4358 & 0.0005 & $\beta \mathrm{H}-7 \rightarrow \beta \mathrm{L}$ & $\mathrm{d}(\mathrm{Cu}) / \mathrm{n}(\mathrm{Br}) / \mathrm{n}(\mathrm{BrAcO}) \rightarrow \mathrm{d}(\mathrm{Cu}) / \sigma^{*}(\mathrm{BrAcO}) / \sigma^{*}(\mathrm{phen})$ & & & \\
\hline & 599.93 & 2.0666 & 0.0008 & $\alpha \mathrm{H}-3 \rightarrow \alpha \mathrm{L}$ & $\mathrm{n}(\mathrm{BrAcO}) / \sigma(\mathrm{BrAcO}) \rightarrow \mathrm{d}(\mathrm{Cu}) / \sigma^{*}(\mathrm{BrAcO}) / \sigma^{*}(\mathrm{phen})$ & & & \\
\hline & 730.15 & 0.0027 & 0.0026 & $\alpha \mathrm{H}-27 \rightarrow \alpha \mathrm{L}$ & $\mathrm{d}(\mathrm{Cu}) / \mathrm{n}(\mathrm{BrAcO}) / \mathrm{n}(\mathrm{Br}) / \sigma($ phen $) \rightarrow \mathrm{d}(\mathrm{Cu}) / \sigma^{*}(\mathrm{BrAcO}) / \sigma^{*}($ phen $)$ & & & \\
\hline
\end{tabular}

Used abbreviations: $\mathrm{d}(\mathrm{Cu})-\mathrm{d}$ orbital of copper cation, $\mathrm{n}(\mathrm{BrAcO})$-non-bonding orbital of bromoacetate anion, $\sigma(\mathrm{BrAcO})-\sigma$ orbital of bromoacetate anion, $\mathrm{n}\left(\mathrm{H}_{2} \mathrm{O}\right)-$ non-bonding orbital of

water, $\mathrm{n}(\mathrm{Br})$-non-bonding orbital of bromide anion, $\pi(\mathrm{phen})-\pi$ orbital of phenanthroline, $\sigma(\mathrm{phen})-\sigma$ orbital of phenanthroline, ${ }^{*}$-an antibonding orbital. 
Three first absorption maxima of the calculated spectrum of 2 are shifted towards larger wavelengths (of around $100 \mathrm{~nm}$ ) comparing to experimental ones. The broad absorption maximum observed above $500 \mathrm{~nm}$ is well reproduced both for 1 and $\mathbf{2}$ (Figure 3, Table 2). Since the studied compounds were treated with UB3LYP, two separate sets of orbitals ( $\alpha$ and $\beta$, Figures 4 and 5) were generated. For both compounds, the electronic transitions involve complex molecular orbitals. Generally, the first two maxima are mostly $n \rightarrow \pi^{*}$ transitions related to ligand-to-ligand charge transfer (LLCT). They involve the non-bonding orbitals of bromoacetate and bromide anions and antibonding $\pi^{*}$ orbitals of phen ligands (Table 2). In a solid-state spectrum of pure phen, those maxima are observed at slightly lower wavelengths, and they are associated with $\pi \rightarrow \pi^{*}$ transitions $[18,21]$. The next absorption maximum is mostly caused by ligand-to-metal charge transfer (LMCT) transitions (Table 2, Figures 4 and 5). The contribution of LMCT seems to be larger in $\mathbf{2}$ (Table 2)-the respective LUMO orbitals involves mostly electrons localized near the metal centers (Figure 5). The corresponding maximum in a solid-state spectrum of pure phen is observed for slightly lower wavelength numbers (Table 2). Similarly to the previous maxima, it is associated with $\pi \rightarrow \pi^{*}$ transitions. The next maximum is related to LLCT and is seen as $n \rightarrow \pi^{*}$ transitions involving lone pair orbitals of bromoacetate and bromide anions, lone pair orbitals of water molecules, and $\pi$ antibonding orbitals of phen (Table 2). For the solid-state spectrum of phen that maximum corresponds to two maxima (Table 2) associated with $n \rightarrow \pi^{*}$ transitions. As other phen maxima, they are observed at smaller wavelengths than the studied compounds' maxima. The last maxima are produced by multiple complex orbital transitions involving $\mathrm{d}$ orbitals of copper cations, non-bonding orbitals of bromoacetic acid/bromoacetate ions, antibonding $\sigma$ orbitals of phen ligands and antibonding $\sigma$ orbitals of bromoacetic acid/bromoacetate ions (Table 2, Figures 4 and 5).

The studied compounds contain a ligand (phen) possessing fluorescence properties in a free state. The number of emitted photons by phen may increase due to changes in the orbitals energy upon coordination. However, the bromine's introduction into the molecular systems diminishes or quenches the fluorescence [37,38]. Both $\mathbf{1}$ and $\mathbf{2}$ show no significant fluorescence, i.e., only a few photons were registered during measurement, and they can be considered instrument artifacts coming from second-order diffraction by grating monochromators (some intensity appears in the area of $\lambda_{\mathrm{em}}=2 \lambda_{\mathrm{ex}}$, Figure S9). Thus, it can be stated that the presence of bromoacetate ions in the studied compounds is a sufficient condition for the total quenching of phen fluorescence. 
(a)
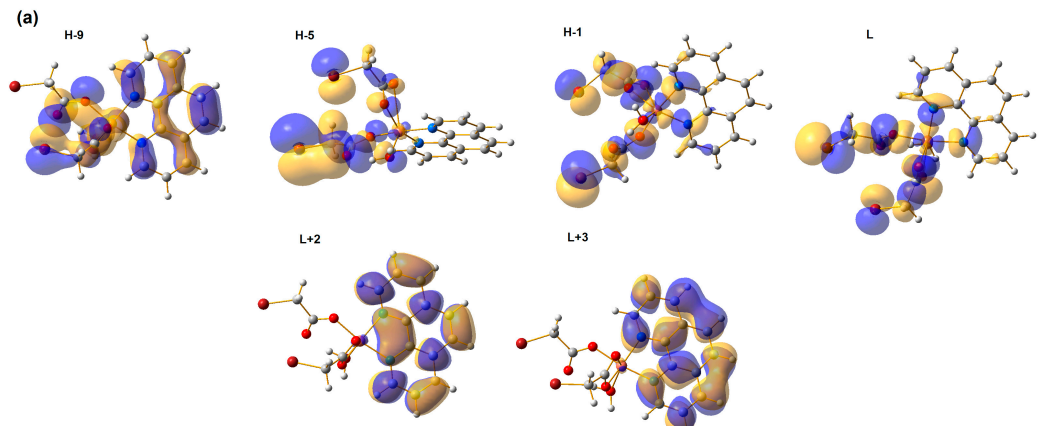

$\llcorner+3$
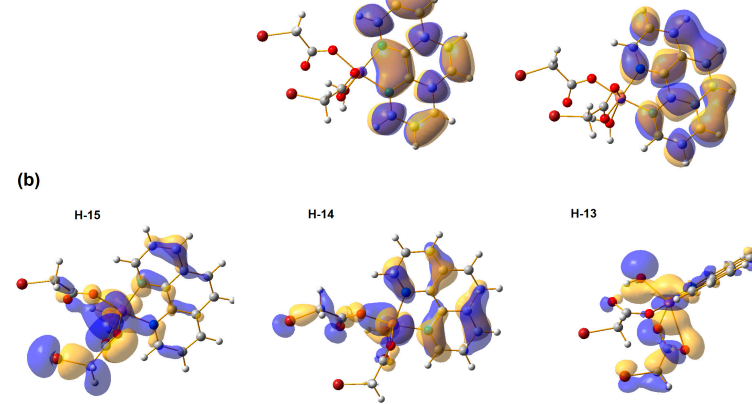

H-13
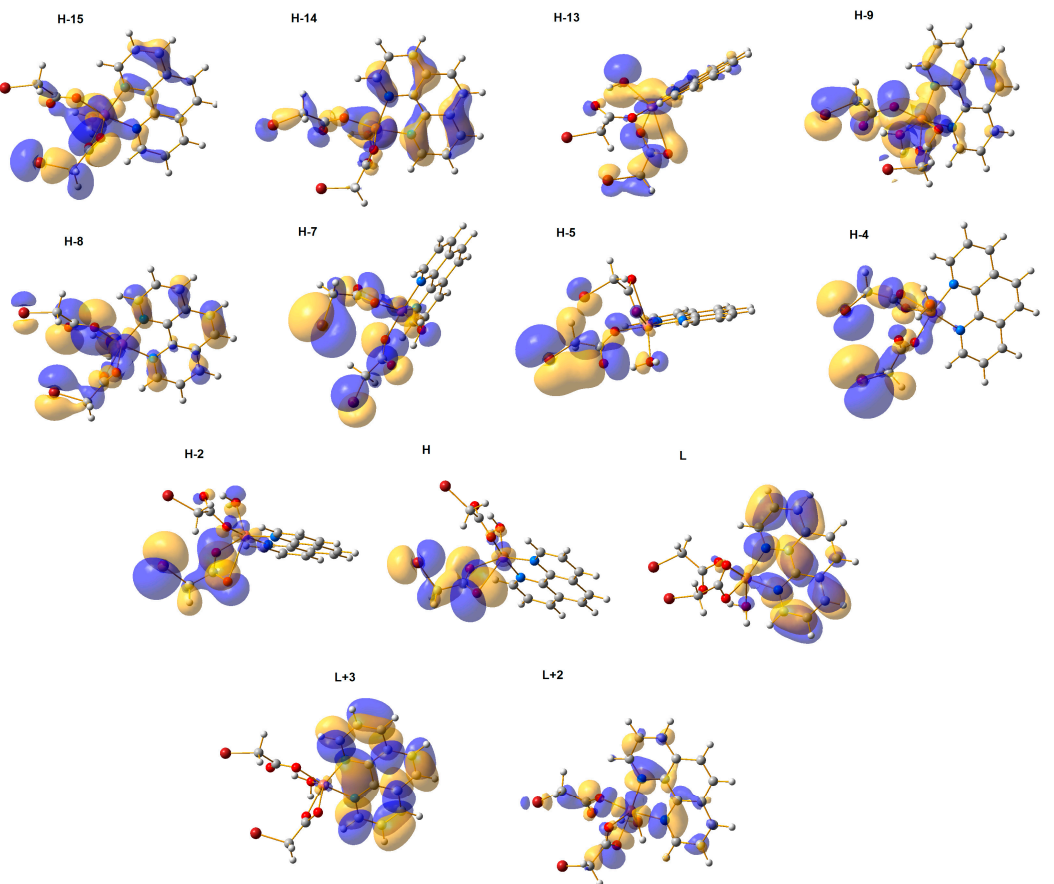

Figure 4. Calculated molecular orbitals of compound 1: $\alpha(\mathbf{a})$ and $\beta(\mathbf{b})$. H letter indicates HOMO, L-LUMO, and \pm (number) represents subsequent orbitals above HOMO and LUMO, respectively. 
(a)
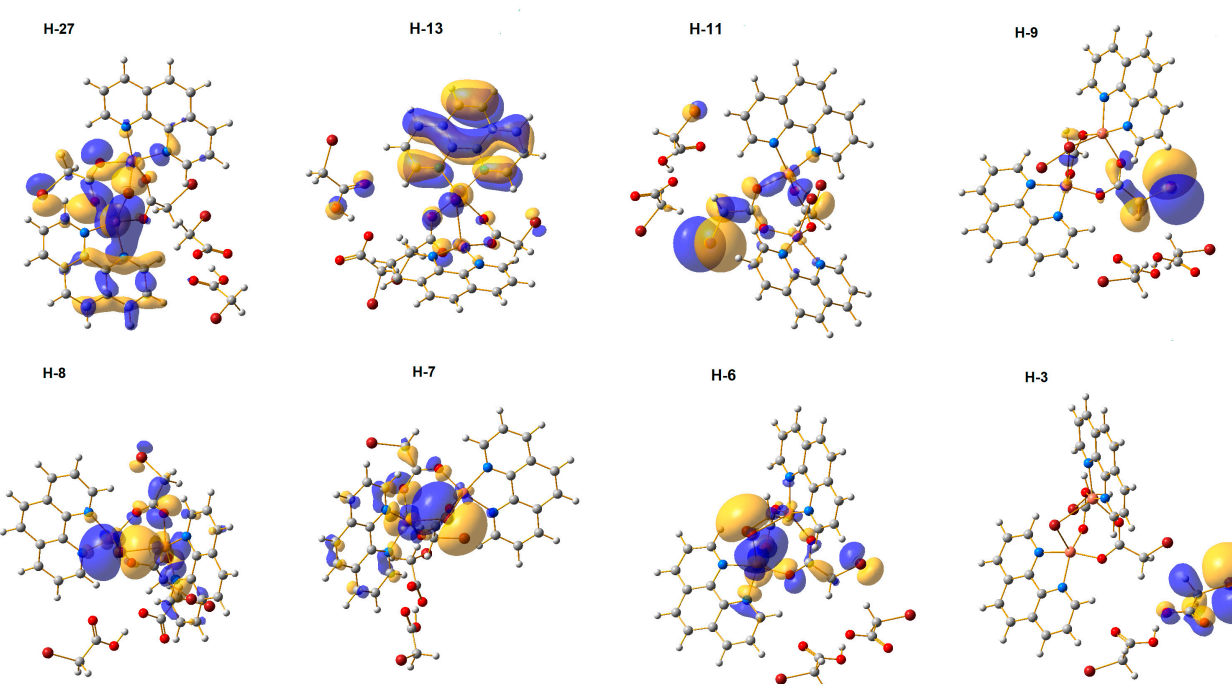

H-7
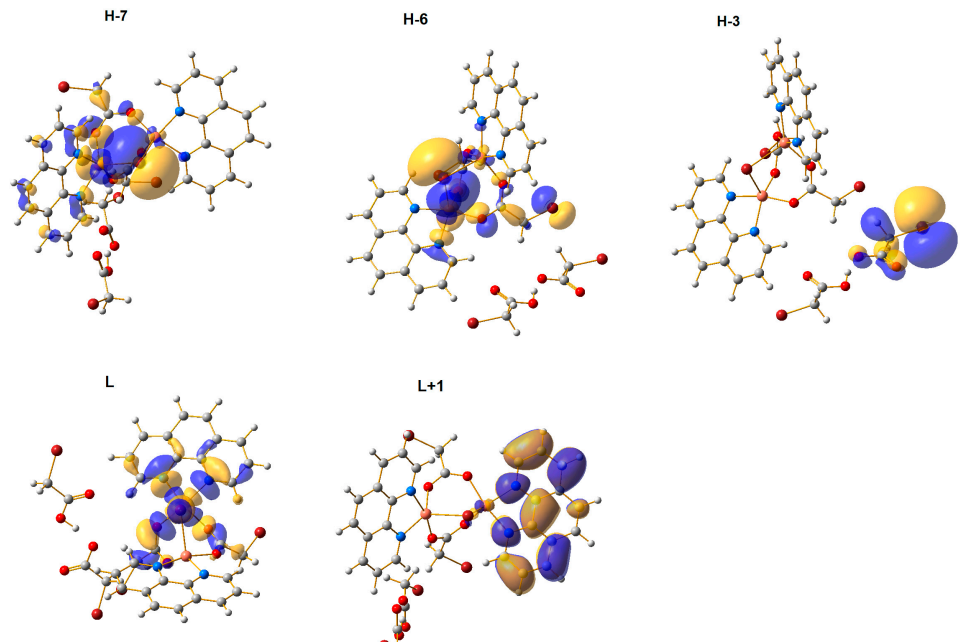

$\mathrm{L}+1$

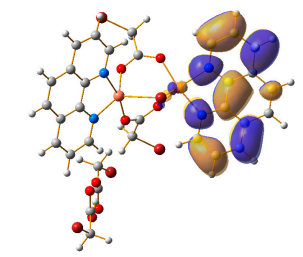

(b)

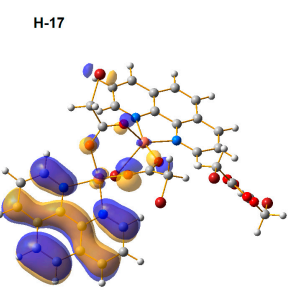

H-15

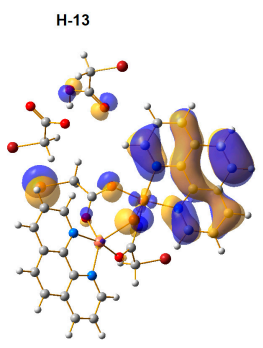

H-8
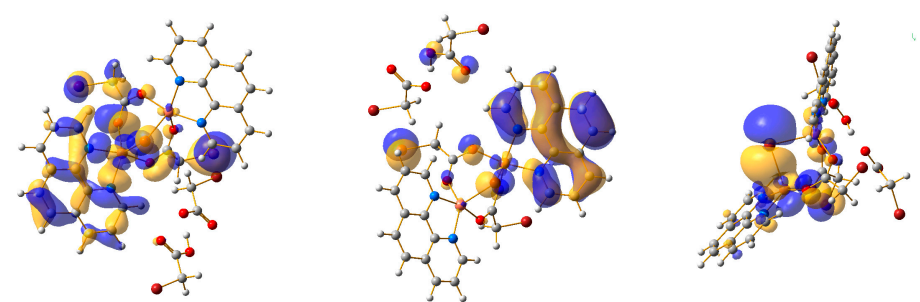

$\mathrm{H}-7$

$H-6$
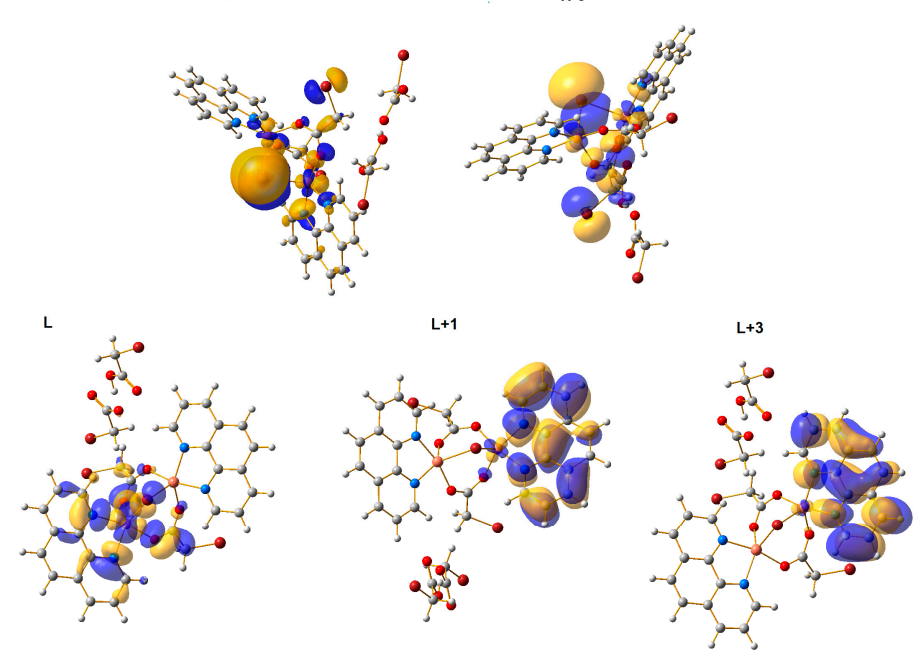

Figure 5. Calculated molecular orbitals of compound 2: $\alpha(\mathbf{a})$ and $\mathrm{B}(\mathbf{b})$. H letter indicates HOMO, L-LUMO, and \pm (number) represents subsequent orbitals above HOMO and LUMO, respectively. 


\subsection{IR Spectra Analysis}

The ATR-IR spectra of the studied compounds contain sets of bands characteristic for bromoacetate anions [39] and phen molecules [40,41] (Table S4, Figure S10). The most important bands of carboxylate anions correspond to stretching vibrations of carboxylate groups. A binding mode of $\mathrm{COO}^{-}$can be estimated based on the separation parameter $\Delta v$, which is a difference between the wavenumbers of $v_{a s}$ $\mathrm{COO}$ and $v_{s} \mathrm{COO}$ [42-44]. In both studied compounds, the bromoacetate anions exhibit two different binding modes, i.e., asymmetric bidentate chelating and monodentate in $\mathbf{1}$, and bidentate syn-syn bridging and non-coordinating in 2. Rarely, in similar cases, it is possible to distinguish two bands of $v_{a s}$, which allow calculation of $\Delta v$ independently for both binding modes of $\mathrm{COO}^{-}$[45]. For the studied compounds, only one $v_{a s}$ band is present, and additionally, it is concealed by the bands of the stretching $\mathrm{CC}$ and $\mathrm{CN}$ vibrations of phen (existing in the same region). In the case of $\mathbf{1}$, the chelating anions exhibit strong asymmetricity of $\mathrm{O}-\mathrm{Cu}$ bond lengths, causing one of these bonds to be significantly stronger than the second. As a result, such chelating mode becomes similar to the monodentate mode, and thus only one $v_{\mathrm{as}}$ band exists. In the structure of $\mathbf{2}$, there are two bridging bromoacetate anions per one non-coordinating anion. Thus the $v_{a s}$ band corresponds very likely to the first ones. The $\Delta v$ values equal to 182 and $204 \mathrm{~cm}^{-1}$, respectively for $\mathbf{1}$ and 2, agree with literature data for the particular binding mode of bromoacetate, i.e., monodentate: $183 \mathrm{~cm}^{-1}$ [46] $191 \mathrm{~cm}^{-1}$ [47] and bidentate syn-syn bridging: $201 \mathrm{~cm}^{-1}$ [48]. The spectrum of 2 confirms the presence of bromoacetic acid in the compound structure. The most evident is the band at $1738 \mathrm{~cm}^{-1}$ produced by the stretching $\mathrm{C}=\mathrm{O}$ vibrations of the carboxylic group [49] (Table S4). Most phen bands are shifted toward larger wavenumbers (compared to pure phen) due to bonds stiffening caused by the formation of a five-membered chelating ring with copper cation. The phen coordination also leads to the differentiation of energy of the same types of oscillators resulting in the splitting of corresponding bands of pure phen, e.g., $v \mathrm{CC}, v \mathrm{CN}, \delta-\alpha \mathrm{CH}$ at $1345 \mathrm{~cm}^{-1}$ or $\delta-\gamma \mathrm{CH}$ at $1092 \mathrm{~cm}^{-1}$ (Table S4).

\subsection{Thermal Analysis}

Compound 1 decomposes in three, well separated, step-processes (Scheme 1, Figure S11). The first stage is dehydration (Figure S12). The dehydrated compound is stable from $128{ }^{\circ} \mathrm{C}$ to $240{ }^{\circ} \mathrm{C}$. In this temperature range, the exothermic process occurs without a mass loss (at $145^{\circ} \mathrm{C}$, Figure S11). It is a transition resulting from structure rearrangement after water evacuation. Next, one of the bromoacetate anion decomposes, and process products are removed. Simultaneously, the second carboxylate undergoes dehalogenation with the formation of glycolate anion and bromide [18]. The last stage comprises the oxidation of glycolate and phen, together with the removal of half of the copper content in the form of copper dibromide, which sublimates at high temperatures [50]. The sublimated $\mathrm{CuBr}_{2}$ decomposes in a mass spectrometer, which is visible in the mass spectrum as characteristic for bromine, $\mathrm{m} / \mathrm{z}$ signals equal to 80 and 160 (Figure S12). The final product is pure copper oxide. The decomposition of 2 begins with the degradation of the non-coordinating bromoacetate, which is less stable than the bromoacetic acid [18,51]. After that stage, the acid's oxidation and the rest of the anions occurs. The phen molecules decompose in the last stage. Similarly to the decomposition of $\mathbf{1}$, the part of the copper content is also removed (Figure S13) and the final product is copper oxide. 


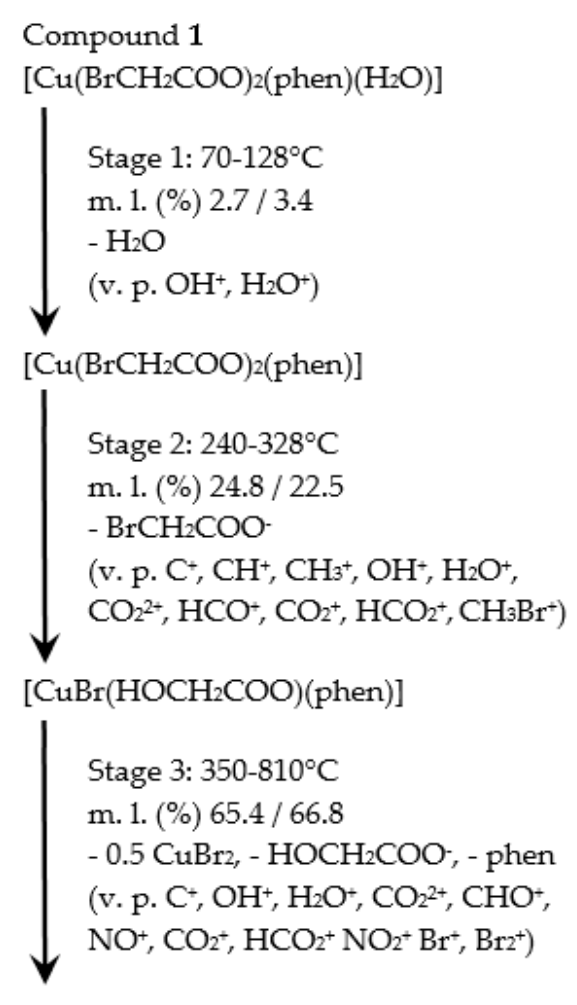

$0.5 \mathrm{CuO}$; m. r. (\%) $7.1 / 7.3$

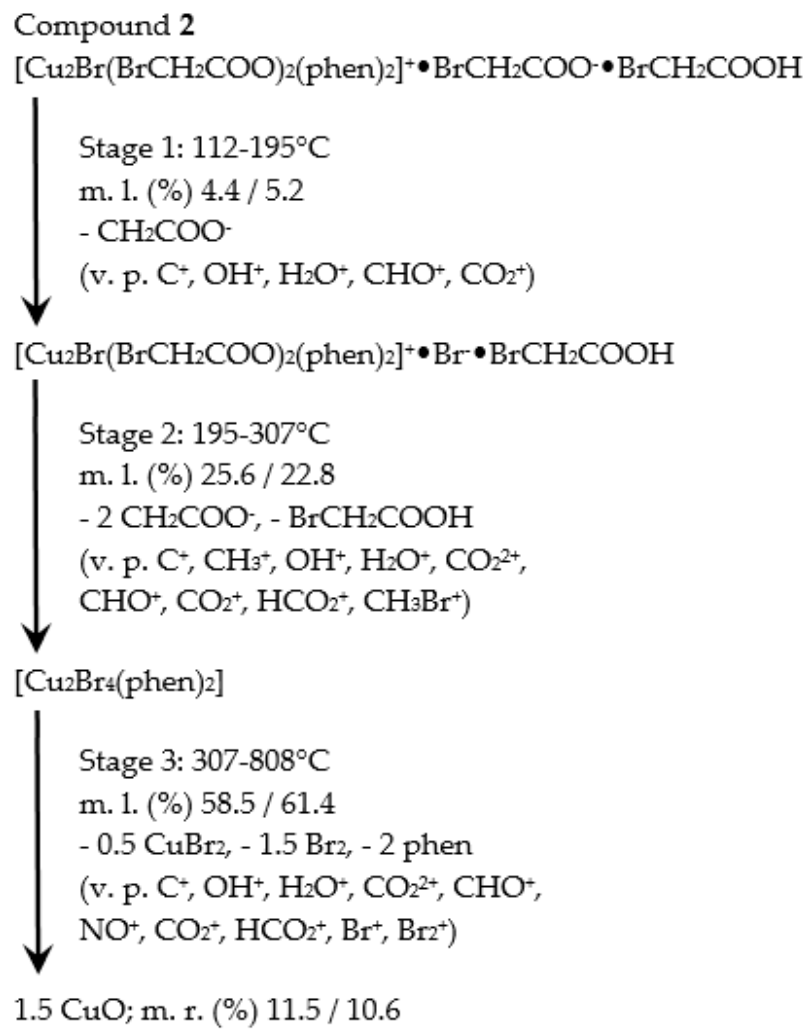

Scheme 1. Thermal decomposition stages of the studied compounds (v. p. = volatile products established from mass spectra; m. 1.—experimental/theoretical mass loss; m. r.-experimental/theoretical mass residue).

\section{Materials and Methods}

\subsection{Synthesis}

All reagents were bought from Sigma-Aldrich (St. Louis, MO, USA) and were analytical grade. The analytical grade water was prepared from improved drinking water via combined reverse osmosis and ion exchange processes.

\subsubsection{Preparation of Copper(II) Bromoacetate Solutions}

Copper(II) bromoacetate solutions was prepared (procedure S1A) by suspending the dicopper carbonate dihydroxide $(0.25 \mathrm{mmol}, 0.0553 \mathrm{~g})$ in a water solution of bromoacetic acid $(0.60 \mathrm{mmol}$, $0.0834 \mathrm{~g}$ of acid dissolved in $5.00 \mathrm{~cm}^{3}$ of water). The mixture was stirred at room temperature (to avoid decomposition of bromoacetate ions) for $30 \mathrm{~min}$ (rotation speed was $300 \mathrm{rpm}$ ). Next, it was filtered (to remove an unreacted excess of dicopper carbonate dihydroxide), and the solid residue was washed with $1.25 \mathrm{~cm}^{3}$ of water. The combined solutions were used in subsequent synthesis. The synthesis was repeated at a larger scale (procedure S2A) with the following alterations of compound/solvent amounts: dicopper carbonate dihydroxide, $2.5 \mathrm{mmol}(0.5528 \mathrm{~g})$; bromoacetic acid, $6.0 \mathrm{mmol}(0.8337 \mathrm{~g})$ dissolved in $25.0 \mathrm{~cm}^{3}$ of water; water used for washing, $5.0 \mathrm{~cm}^{3}$.

\subsubsection{Synthesis of Coordination Compounds}

The coordination compounds were synthesized in a reaction between the copper(II) bromoacetate and 1,10-phenanthroline (phen). The 2:1 molar ratio of metal to ligand (M:L) was applied. The above described (procedure S1A) water solution of copper bromoacetate $\left(0.3 \mathrm{mmol}, 6.25 \mathrm{~cm}^{3}\right)$ was mixed with the solution of phen $\left(0.15 \mathrm{mmol}, 0.0297 \mathrm{~g}\right.$ dissolved in $5.0 \mathrm{~cm}^{3}$ equivolume mixture of methanol and water; procedure S1B). The resulting solution was stirred vigorously (rotation speed was $1500 \mathrm{rpm}$ ) 
at room temperature for $30 \mathrm{~min}$ and it was left to crystallize at $20{ }^{\circ} \mathrm{C}$. Light blue prism crystals of $\left[\mathrm{Cu}\left(\mathrm{BrCH}_{2} \mathrm{COO}\right)_{2}(\right.$ phen $\left.)\left(\mathrm{H}_{2} \mathrm{O}\right)\right](1)$ grew after one week (mass: $0.0742 \mathrm{~g}$, yield $($ phen) $=92 \%$ ). The synthesis was repeated at a larger scale (procedure S2B) with the following alterations: the water solution of copper bromoacetate $\left(3.0 \mathrm{mmol}, 30 \mathrm{~cm}^{3}\right)$ from procedure S2A was used, and the solution of phen contained $1.5 \mathrm{mmol}, 0.2973 \mathrm{~g}$ of the substance dissolved in $10.0 \mathrm{~cm}^{3}$ of solvent mixture. Deep blue plate crystals of $\left.\left[\mathrm{Cu}_{2} \mathrm{Br}\left(\mathrm{BrCH}_{2} \mathrm{COO}\right)_{2} \text { (phen }\right)_{2}\right]^{+} \mathrm{BrCH}_{2} \mathrm{COO}^{-} \mathrm{BrCH}_{2} \mathrm{COOH}$ (2) formed after 3 days (mass: $0.4117 \mathrm{~g}$, yield $_{(\text {phen })}=49 \%$ ) were separated by filtration and after one week the light blue prism crystals of 1 were formed (mass: $0.3387 \mathrm{~g}$, $\operatorname{yield}_{(\text {phen) }}=42 \%$ ). Elemental analyses included C, H, N, O elements were carried out using Vario EL III CHNOS Elemental Analyzer (Elementar, Langenselbold, Germany). Results for 1 [calculated/found (\%)]: C 35.74/35.36; H 2.62/2.31; N 5.21/5.09; O 14.88/15.02 and for 2: C 34.31/34.01; H 2.25/2.11; 5.00/4.88; O 11.43/11.54.

\subsection{Crystal Structure Determination}

X-ray intensity data of the studied compounds were collected at 100.0(1) K, on a Rigaku Synergy Dualflex automatic diffractometer (Rigaku Corporation, Tokyo, Japan) equipped with Pilatus 300K detector and micro-focus sealed PhotonJet $X$-ray tubes generated monochromated $\operatorname{MoK}_{\alpha}(\lambda=0.71073$ $\AA)$ or $C u K_{\alpha}(\lambda=1.54184 \AA)$ radiation, with shutterless $\omega$ scan mode (the optimal radiation wavelength was selected according to preliminary measurement results performed for both wavelengths: $\mathrm{Cu} K_{\alpha}$ for 1 and $\mathrm{MoK}_{\alpha}$ for 2). Lorentz, polarization, and empirical absorption correction (using spherical harmonics, implemented in SCALE3 ABSPACK scaling algorithm) corrections were applied during the data reduction. The structure was solved by dual-space algorithm. All non-hydrogen atoms were refined anisotropically using full-matrix, least-squares technique on $F^{2}$. All hydrogen atoms were found from difference Fourier synthesis after ten cycles of anisotropic refinement. Carbon bonded hydrogen atoms were refined as "riding" on the adjacent atom with geometric idealization after each cycle of refinement. Individual isotropic displacement factors of carbon bonded $\mathrm{H}$ atoms were set to be equal to 1.2 times the value of equivalent displacement factors of the parent atoms. Oxygen bonded hydrogen atoms positions were freely refined, and their individual isotropic displacement factors were fixed to 1.5 of the value of equivalent displacement factors of the parent atoms. For compound 2, the occupancy factor of the carboxylic group hydrogen atom $(\mathrm{H} 22 \mathrm{O})$ was set to 0.50 as the $\mathrm{O} 22-\mathrm{H} 22 \mathrm{O}$ group interacts with the symmetry generated $\mathrm{O} 22(-\mathrm{x}+0.5,-\mathrm{y}+0.5,-\mathrm{z}+1)$ atom and thus, the $\mathrm{H}$ atom can exist only at one of these $\mathrm{O} 22$ atoms. Thus, the $\mathrm{H}$ atom is equally disordered by symmetry over two positions and exists either in the asymmetric unit or as the symmetry generated $\mathrm{H}$ atom. Existence in both positions (i.e., at both neighboring molecules) is impossible due to the formation of an unacceptably short $\mathrm{H} 22 \mathrm{O} \bullet \bullet \bullet \mathrm{H} 22 \mathrm{O}(-\mathrm{x}+0.5,-\mathrm{y}+0.5,-\mathrm{z}+1)$ distance. The SHELXT [52], SHELXL [53] and SHELXTL [54] programs were used for all calculations. Atomic scattering factors were taken from International Tables for Crystallography [55]. Details concerning crystal data and refinement are given in Table 3.

CCDC 1990904 (compound 1) and 1990905 (compound 2) contain the supplementary crystallographic data for this paper. These data can be obtained free of charge via http://www. ccdc.cam.ac.uk/conts/retrieving.html (or from the CCDC, 12 Union Road, Cambridge CB2 1EZ, UK; Fax: +44-1223-336033; E-mail: deposit@ccdc.cam.ac.uk) 
Table 3. Crystal data and structure refinement details for the studied compounds.

\begin{tabular}{|c|c|c|}
\hline Compound & 1 & 2 \\
\hline Empirical formula & $\mathrm{C}_{16} \mathrm{H}_{14} \mathrm{Br}_{2} \mathrm{CuN}_{2} \mathrm{O}_{5}$ & $\mathrm{C}_{32} \mathrm{H}_{25} \mathrm{Br}_{5} \mathrm{Cu}_{2} \mathrm{~N}_{4} \mathrm{O}_{8}$ \\
\hline Formula weight & 537.65 & 1120.19 \\
\hline Crystal system & Triclinic & Monoclinic \\
\hline Space group & $P-1$ (No. 2) & C2/c (No. 15) \\
\hline \multicolumn{3}{|l|}{ Unit cell dimensions } \\
\hline $\mathrm{a}(\AA)$ & $9.1666(2)$ & $25.3799(7)$ \\
\hline $\mathrm{b}(\AA)$ & $10.4887(2)$ & $7.2326(2)$ \\
\hline$c(\AA)$ & $11.0716(3)$ & $20.1769(5)$ \\
\hline$\alpha\left(^{\circ}\right)$ & $65.382(2)$ & 90.00 \\
\hline$\beta\left(^{\circ}\right)$ & $66.608(2)$ & 107.993(3) \\
\hline$\gamma\left({ }^{\circ}\right)$ & $73.039(2)$ & 90.00 \\
\hline Volume $\left(\AA^{3}\right)$ & 877.95(4) & $3522.59(17)$ \\
\hline $\mathrm{Z}$ & 2 & 4 \\
\hline Calculated density $\left(\mathrm{Mg} / \mathrm{m}^{3}\right)$ & 2.034 & 2.112 \\
\hline Absorption coefficient $\left(\mathrm{mm}^{-1}\right)$ & 7.371 & 6.918 \\
\hline$F(000)$ & 526 & 2168 \\
\hline Crystal size $(\mathrm{mm})$ & $0.060 \times 0.095 \times 0.179$ & $0.016 \times 0.038 \times 0.242$ \\
\hline$\theta$ Range for data collection $\left(^{\circ}\right)$ & 4.632 to 78.742 & 2.940 to 31.436 \\
\hline & $-11 \leq \mathrm{h} \leq 11$ & $-36 \leq \mathrm{h} \leq 34$ \\
\hline Index ranges & $\begin{array}{l}-13 \leq \mathrm{k} \leq 12 \\
-13 \leq 1 \leq 13\end{array}$ & $\begin{array}{l}-10 \leq \mathrm{k} \leq 10 \\
-28 \leq 1 \leq 26\end{array}$ \\
\hline Reflections collected / unique & $40793 / 3603$ & $30256 / 5249$ \\
\hline $\mathrm{R}_{i n t}$ & 0.0307 & 0.0471 \\
\hline Completeness $(\%)$ & $99.9\left(\right.$ to $\left.\theta=67^{\circ}\right)$ & $99.9\left(\right.$ to $\left.\theta=25^{\circ}\right)$ \\
\hline Min. and max. transmission & 0.500 and 0.973 & 0.404 and 1.000 \\
\hline Data / restraints / parameters & $3603 / 0 / 241$ & $5249 / 0 / 234$ \\
\hline Goodness-of-fit on $F^{2}$ & 1.083 & 1.013 \\
\hline Final $R$ indices $[I>2 \sigma(I)]$ & $\begin{array}{r}R 1=0.0211 \\
w R 2=0.0535\end{array}$ & $\begin{array}{r}R 1=0.0320 \\
w R 2=0.0756\end{array}$ \\
\hline $\mathrm{R}$ indices (all data) & $\begin{array}{r}R 1=0.0215 \\
w R 2=0.0537\end{array}$ & $\begin{array}{r}R 1=0.0448 \\
w R 2=0.0802\end{array}$ \\
\hline $\begin{array}{l}\text { Largest diff. peak and hole } \\
\qquad\left(\mathrm{e} \bullet \AA^{-3}\right)\end{array}$ & 0.846 and -0.917 & 1.869 and -1.368 \\
\hline
\end{tabular}

\subsection{Other Physical Measurements}

The UV-Vis diffuse reflectance spectra were recorded on a Jasco V-660 spectrometer (Jasco, Easton, MD, USA), in the spectral range $200-850 \mathrm{~nm}$, using spectralon [56] as a standard with $100 \%$ reflectance. The three-dimensional fluorescence spectra were recorded on a Jasco FP-6300 spectrofluorometer (Jasco, Easton, MD, USA), with solid samples directed at an angle of $30^{\circ}$ to the incident beam. The excitation and emission wavelength ranges were $220-640 \mathrm{~nm}$ and $230-740 \mathrm{~nm}$, respectively. The data pitch and bandwidth were $1 \mathrm{~nm}$ on both monochromators. The ATR-IR spectra of the coordination compounds were recorded on a Bruker INVENIO-R spectrometer (Bruker Optik GmbH, Ettlingen, Germany) in the spectral range $4000-400 \mathrm{~cm}^{-1}$. The thermal analyses were carried out with a Netzsch STA 449 F1 Jupiter thermoanalyzer (Netzsch-Geratebau GmbH, Selb, Germany) coupled with a Netzsch Aeolos Quadro QMS 403 mass spectrometer (Netzsch-Geratebau GmbH, Selb, Germany). Samples were heated in corundum crucibles up to $1000^{\circ} \mathrm{C}$, with a heating rate $10^{\circ} \mathrm{C} \cdot \mathrm{min}^{-1}$ in synthetic air $\left(20 \% \mathrm{O}_{2}, 80 \% \mathrm{~N}_{2}\right)$ flow.

\subsection{Quantum-Mechanical Calculations}

The excited states of the studied coordination compounds have been calculated for X-ray determined coordinates using TD-DFT method. Input structural models were prepared with Mercury CSD 4.3.0 [57] computer program (Cambridge Crystallography Data Centre, Cambridge, 
UK). Positions of hydrogen atoms have been normalized by moving them along the covalent bond vector $(\mathrm{X} \rightarrow \mathrm{H})$ to the $\mathrm{X}-\mathrm{H}$ distance equal to the average neutron diffraction value. In each case, as an input, a coordination unit of a respective compound was used. For 2, the species occupying the outer coordination sphere were included. All calculations were performed utilizing Gaussian09 rev. D.01 (Gaussian Inc., Wallingford, CT, USA) [58] with B3LYP functional and employing 6-31++g(2d,2p) basis set of Pople et al. [36,59]. The absorption maxima of 2 were not accurately reproduced treated the whole system as a singlet or triplet state. Hence, it was calculated using unrestricted DFT (UB3LYP) to model the antiferromagnetic coupling of two $\mathrm{Cu}(\mathrm{II})$ doublet state centers (with $\alpha$ and $\beta$ spins respectively so that all the $\alpha$ electrons could cancel all of the $\beta$ ones). To ensure that the open singlet state system was the one with the lowest energy, the calculation was repeated using the wave function's optimization. This process led to a stable wave function and further decreased the system energy (Table S3). The resulted electronic state was then used in TD-DFT calculation. The number of calculated transitions was set to 130 to cover all the experimentally founded bands. The calculated excited states' assignment to the observed experimental maxima was based on the comparison of excitation energies and the oscillator strengths/intensities of the corresponding maxima. The analysis of the character of respective orbital excitations was based on orbital contour plots. Hirshfeld surface maps and the studied compounds' finger plots were generated using Crystal Explorer 17.5 [60].

\section{Conclusions}

Two structurally different coordination compounds were synthesized in the reaction between copper bromoacetate and phen used in 2:1 molar ratio. The formation of these compounds is dependent on substrates concentrations (ion strength of solution). Compound $\mathbf{2}$ is possible to obtain only above a specific concentration, for which ion strength is large enough to decompose bromoacetates and release bromides to the solution. The structural diversity of the studied compounds causes dissimilarities of spectral and thermal properties. This work also highlights the difficulties that may be encountered while analyzing the spectroscopic properties of open-shell systems. Dealing with a system in which one would assume some open shell character checking wave function stability should always be considered. Treatment of the system as open-shell, without checking the wave function stability, does not always allow to obtain a system with the lowest energy.

Supplementary Materials: The following are available online. Supplemental experimental section, Supplemental discussion, Table S1: Hydrogen bonds in the studied compounds. Table S2: Stacking interactions in the studied compounds. Table S3: The energy of 2 calculated for its various spin states. Table S4: Vibrational frequencies and their assignments for the studied compounds. Figures S1-S5: Hirshfeld surfaces and 2D decomposition fingerprints of the studied compounds. Figure S6: Stacking interactions in the studied compounds. Figures S7-S8: Hirshfeld surfaces and 2D decomposition fingerprints of literature known compound (CSD refcode: NOQPEP). Figure S9: Three-dimensional fluorescence spectra of the studied compounds. Figure S10: ATR-IR spectra of the studied compounds. Figure S11: TG, DTA, and DTG curves for the studied compounds. Figure S12-S13: Mass spectra of volatile products from the thermal decomposition of the studied compounds. Figure S14: Experimental and calculated UV-Vis spectra of 2.

Author Contributions: Investigation, writing-original draft preparation, E.K.; methodology, investigation, writing-original draft preparation, writing-review \& editing, visualization, T.S.; methodology, investigation, writing-original draft preparation, writing-review \& editing, visualization, M.Ś.; investigation, writing-original draft preparation, writing-review \& editing, M.B.; conceptualization, writing-original draft preparation, funding acquisition, R.K. All authors have read and agreed to the published version of the manuscript.

Funding: This work was financed by funds allocated by the Ministry of Science and Higher Education (Poland) to the Institute of General and Ecological Chemistry, Lodz University of Technology.

Acknowledgments: The calculations mentioned in this paper are performed using TUL Computing \& Information Services Center infrastructure.

Conflicts of Interest: The authors declare no conflict of interest. 


\section{References}

1. Masternak, J.; Zienkiewicz-Machnik, M.; Kowalik, M.; Jabłońska-Wawrzycka, A.; Rogala, P.; Adach, A.; Barszcz, B. Recent advances in coordination chemistry of metal complexes basedon nitrogen heteroaromatic alcohols. Synthesis, structures andpotential applications. Coord. Chem. Rev. 2016, 327-328, 242-270. [CrossRef]

2. Rasheed, T.; Nabeel, F. Luminescent metal-organic frameworks as potential sensory materials for various environmental toxic agents. Coord. Chem. Rev. 2019, 401, 213065. [CrossRef]

3. Petricek, S. Structural diversity of nickel and manganese chloride complexes with pyridin-2-one. Molecules 2020, 25, 846. [CrossRef] [PubMed]

4. Swiatkowski, M.; Kruszynski, R. Revealing the structural chemistry of the group 12 halide coordination compounds with 2,2'-bipyridine and 1,10-phenanthroline. J. Coord. Chem. 2017, 70, 642-675. [CrossRef]

5. Liu, K.; Shi, W.; Cheng, P. The coordination chemistry of $\mathrm{Zn}(\mathrm{II}), \mathrm{Cd}(\mathrm{II})$ and $\mathrm{Hg}(\mathrm{II})$ complexes with 1,2,4-triazole derivatives. Dalton Trans. 2011, 40, 8475-8490. [CrossRef] [PubMed]

6. Walfort, B.; Auth, T.; Degel, B.; Helten, H.; Stalke, D. Copper and Silver Triimidosulfites: S(NtBu)32-Bicapped M3-Triangles Connected via Lithium Halide Ladders or Fragments Thereof. Organometallics 2002, 21, 2208-2214. [CrossRef]

7. Herber, U.; Moegling, J.; Siris, R.; Hoffmann, A.; Mayer, P.; Göbel, C.; Lochenie, C.; Weber, B.; Herres-Pawlis, S. A Study on FeII, ZnII and CuII Complexes with Novel Tridentate Bis(pyrazolyl)methane Ligands. Anorg. Allg. Chem. 2018, 644, 1576-1592. [CrossRef]

8. Dhayal, R.S.; Liao, J.-H.; Wang, X.; Liu, Y.-C.; Chiang, M.-H.; Kahlal, S.; Saillard, J.-Y.; Liu, C.W. Diselenophosphate-Induced Conversion of an Achiral [Cu20H11\{S2P(OiPr)2\}9] into a Chiral [Cu20H11\{Se2P(OiPr)2\}9] Polyhydrido Nanocluster. Angew. Chem. Int. Ed. 2015, 54, 13604-13608. [CrossRef]

9. Levason, W.; Spicer, M.D. The chemistry of copper and silver in their higher oxidation states. Coord. Chem. Rev. 1987, 76, 45-120. [CrossRef]

10. Popova, T.V.; Aksenova, N.V. Complexes of Copper in Unstable Oxidation States. Russ. J. Coord. 2003, 29, 743-765. [CrossRef]

11. Goswami, A.; Schmittel, M. Heteroleptic copper phenanthroline complexes in motion: From stand-alone devices to multi-component machinery. Coord. Chem. Rev. 2018, 376, 478-505. [CrossRef] [PubMed]

12. Trose, M.; Nahra, F.; Cazin, C.S.J. Dinuclear N-heterocyclic carbene copper(I) complexes. Coord. Chem. Rev. 2018, 355, 380-403. [CrossRef]

13. Dogan, S.; Mutlu Balci, C.; Senocak, A.; Besli, S. Cu(II) complexes of cyclotriphosphazene bearing Schiff bases: Synthesis, structural characterization, DFT calculations, absorbance and thermal properties. Polyhedron 2020, 183, 114541. [CrossRef]

14. Iqbal, M.; Karim, A.; Ali, S.; Nawaz Tahir, M.; Sohail, M. Synthesis, characterization, structural elucidation, electrochemistry, DNA binding study, micellization behaviour and antioxidant activity of the $\mathrm{Cu}(\mathrm{II})$ carboxylate complexes. Polyhedron 2020, 178, 114310. [CrossRef]

15. Davidovich, R.L.; Stavila, V.; Marinin, D.V.; Voit, E.I.; Whitmire, K.H. Stereochemistry of lead(II) complexes with oxygen donor ligands. Coord. Chem. Rev. 2009, 253, 1316-1352. [CrossRef]

16. Irto, A.; Cardiano, P.; Cataldo, S.; Chand, K.; Cigala, R.M.; Crea, F.; De Stefano, C.; Gattuso, G.; Muratore, N.; Pettignano, A.; et al. Speciation studies of bifunctional 3-hydroxy-4-pyridinone ligands in the presence of $\mathrm{Zn}^{2+}$ at different ionic strengths and temperatures. Molecules 2019, 24, 4084. [CrossRef]

17. Urbansky, E.T. The Fate of the Haloacetates in Drinking Water Chemical Kinetics in Aqueous Solution. Chem. Rev. 2001, 101, 3233-3244. [CrossRef]

18. Kruszynski, R. Synthesis of coordination compounds via dehalogenation of zinc bromoacetate in presence of some amines. Inorg. Chim. Acta 2011, 371, 111-123. [CrossRef]

19. Gao, S.; Huang, M.; Sun, Z.; Li, D.; Xie, C.; Feng, L.; Liu, S.; Zheng, K.; Pang, Q. A new mixed-ligand lanthanum(III) complex with salicylic acid and 1,10-phenanthroline: Synthesis, characterization, antibacterial activity, and underlying mechanism. J. Mol. Struct. 2021, 1225, 129096. [CrossRef]

20. Aycan, T.; Ozturk, F.; Doruk, T.; Demir, S.; Fidan, M.; Pasaoglu, H. Synthesis, structural, spectral and antimicrobial activity studies of copper-nalidixic acid complex with 1,10-phenanthroline: DFT and molecular docking. Spectrochim. Acta A 2020, 241, 118639. [CrossRef] 
21. Swiatkowski, M.; Kruszynski, R. Structural insights into the usage of carboxylate ions as molecular pins. Polyhedron 2017, 135, 265-277. [CrossRef]

22. Golovnev, N.N.; Molokeev, M.S.; Sterkhova, I.V.; Lesnikov, M.K.; Demina, A.V.; Patrin, G.S. Two new Cu(II) and $\mathrm{Ni}$ (II) 1,10-phenanthroline complexes with anions of barbituric acids in the outer sphere: Synthesis, structure, spectroscopic, magnetic and thermal properties. J. Mol. Struct. 2020, 1219, 128526. [CrossRef]

23. Onawumi, O.O.; Adekunle, F.A.; Ibrahim, A.O.; Rajasekharan, M.V.; Odunola, O.A. Synthesis, Characterization, and Crystal Structures of $\left[\mathrm{Cu}(\text { phen })_{2} \mathrm{Cl}\right] \mathrm{Cl} .6 .5 \mathrm{H}_{2} \mathrm{O}$ and $\left[\mathrm{Cu}(\mathrm{phen})_{2} \mathrm{Br}\right] \mathrm{Br}$. Synth. React. Inorg. M. 2010, 40, 78-83.

24. Inczedy, J. Równowagi kompleksowania w chemii analitycznej, (eng. Analytical Application of Complex Equilibria); PWN: Warsaw, Poland, 1979; p. 263.

25. He, G.; Sun, J.; Zhao, R.; Li, J. Bis(2-bromoacetato- $\left.\kappa^{2} \mathrm{O}, \mathrm{O}^{\prime}\right)\left(1,10\right.$-phenanthroline- $\left.\kappa^{2} \mathrm{~N}, \mathrm{~N}^{\prime}\right) \operatorname{copper}(\mathrm{II})$. Acta Crystallogr. Sect. E Struct. Rep. Online 2009, 65, m417. [CrossRef] [PubMed]

26. Groom, C.R.; Bruno, I.J.; Lightfoot, M.P.; Ward, S.C. Cambridge Structural Database, CSD v 5.39, August 2018. Acta Crystallogr. Sect. B Struct. Sci. 2016, 72, 171-179. [CrossRef] [PubMed]

27. Kepert, D.L. Aspects of the Stereochemistry of Six-coordination. In Progress in Inorganic Chemistry; Lippard, S.J., Ed.; John Wiley: New York, NY, USA, 1977; Volume 23.

28. Batsanov, S.S. Van der Waals Radii of Elements. Inorg. Mater. 2001, 37, 871-885. [CrossRef]

29. Hu, S.-Z.; Zhou, Z.-H.; Robertson, B.E. Consistent approaches to van der Waals radii for the metallic elements. Z. Kristallogr. 2009, 224, 375-383. [CrossRef]

30. Zachariasen, W.H. Bond lengths in oxygen and halogen compounds of $\mathrm{d}$ and $\mathrm{f}$ elements. J. Less-Common Met. 1978, 62, 1-7. [CrossRef]

31. Brown, I.D. Influence of Chemical and Spatial Constraints on the Structures of Inorganic Compounds. Acta Crystallogr. Sect. B Struct. Sci. 1997, 53, 381-393. [CrossRef]

32. Brown, I.D. Chemical and steric constraints in inorganic solids. Acta Crystallogr. Sect. B Struct. Sci. 1992, 48, 553-572. [CrossRef]

33. Shields, P.G.; Raithby, P.R.; Allen, F.H.; Motherwell, W.D.S. The assignment and validation of metal oxidation states in the Cambridge Structural Database. Acta Crystallogr. Sect. B Struct. Sci. 2000, 56, 455-465. [CrossRef] [PubMed]

34. Favas, M.C.; Kepert, D.L. Aspects of the Stereochemistry of Four-Coordination and Five-Coordination. In Progress in Inorganic Chemistry; Lippard, S.J., Ed.; John Wiley: New York, NY, USA, 1980; Volume 27, pp. 325-463.

35. Ortega, G.; Hernández, J.; González, T.; Dorta, R.; Briceño, A. 1,2,4,5-Benzenetetracarboxylic acid: A versatile hydrogen bonding template for controlling the regioselective topochemical synthesis of head-to-tail photodimers from stilbazole derivatives. Photochem. Photobiol. Sci. 2018, 17, 670-680. [CrossRef] [PubMed]

36. Gordon, M.S.; Binkley, J.S.; Pople, J.A.; Pietro, W.J.; Hehre, W.J. Self-consistent molecular-orbital methods. 22. Small split-valence basis sets for second-row elements. J. Am. Chem. Soc. 1982, 104, 2797-2803. [CrossRef]

37. Shao, Y.; Zhang, X.; Liang, K.; Wang, J.; Lin, Y.; Yang, S.; Zhang, W.-B.; Zhu, M.; Sun, B. How does the interplay between bromine substitution at bay area and bulky substituents at imide position influence the photophysical properties of perylene diimides? RSC Adv. 2017, 7, 16155-16162. [CrossRef]

38. Chen, P.; Ge, F.; Cai, Z. Effects of halogen substitution and solvent on the fluorescent spectra of fluorescein. Chem. Bullet./Huaxue Tongbao 2014, 77, 243-249.

39. Katon, J.E.; Kleinlein, R.L. The vibrational spectra and structure of sodium bromoacetate and sodium bromoacetate-d $\mathrm{d}_{2}$. J. Mol. Struct. 1973, 17, 239-248. [CrossRef]

40. Reiher, M.; Brehm, G.; Schneider, S. Assignment of Vibrational Spectra of 1,10-Phenanthroline by Comparison with Frequencies and Raman Intensities from Density Functional Calculations. J. Phys. Chem. A 2004, 108, 734-742. [CrossRef]

41. Muniz-Miranda, M. Surface Enhanced Raman Scattering and Normal Coordinate Analysis of 1,10-Phenanthroline Adsorbed on Silver Sols. J. Phys. Chem. A 2000, 104, 7803-7810. [CrossRef]

42. Deacon, G.B.; Phillips, R.J. Relationships between the carbon-oxygen stretching frequencies of carboxylato complexes and the type of carboxylate coordination. Coord. Chem. Rev. 1980, 33, 227-250. [CrossRef]

43. Zelenak, V.; Vargova, Z.; Gyoryova, K. Correlation of infrared spectra of zinc(II) carboxylates with their structures. Spectrochim. Acta A 2007, 66, 262-272. [CrossRef] 
44. Sutton, C.C.R.; da Silva, G.; Franks, G.V. Modeling the IR Spectra of Aqueous Metal Carboxylate Complexes: Correlation between Bonding Geometry and Stretching Mode Wavenumber Shifts. Chem. A Eur. J. 2015, 21, 6801-6805. [CrossRef] [PubMed]

45. Swiatkowski, M.; Kruszynski, R. Structurally diverse coordination compounds of zinc as effective precursors of zinc oxide nanoparticles with various morphologies. Appl. Organomet. Chem. 2019, 33, e4812. [CrossRef]

46. Sharutin, V.V.; Sharutina, O.K.; Efremov, A.N.; Andreev, P.V. Synthesis and Structures of Tris(3-Fluorophenyl) antimony Dicarboxylates: $\left(3-\mathrm{FC}_{6} \mathrm{H}_{4}\right)_{3} \mathrm{Sb}[\mathrm{OC}(\mathrm{O}) \mathrm{R}]_{2}\left(\mathrm{R}=\mathrm{C}_{6} \mathrm{H}_{3}\left[\left(\mathrm{NO}_{2}\right)_{2}-3,5\right)\right], \mathrm{CH}_{2} \mathrm{Br}_{,} \mathrm{CH}_{2} \mathrm{Cl}$, and $\left.\mathrm{CH}=\mathrm{CHPh}\right)$. Russ. J. Coord. Chem. 2018, 44, 635-641. [CrossRef]

47. Sharutin, V.V.; Sharutina, O.K.; Efremov, A.N. Tri-para-Tolylantimony Dicarboxylates (4-MeC6H4)3Sb $[\mathrm{OC}(\mathrm{O}) \mathrm{R})] 2, \mathrm{R}=\mathrm{C} 6 \mathrm{H} 4(\mathrm{NO} 2-3), \mathrm{C} 6 \mathrm{H} 3(\mathrm{NO} 2) 2-3,5, \mathrm{CH} 2 \mathrm{Br}$ : Synthesis and Structure. Russ. J. Inorg. Chem. 2019, 64, 68-73. [CrossRef]

48. Uchida, S.; Eguchi, R.; Nakamura, S.; Ogasawara, Y.; Kurosawa, N.; Mizuno, N. Selective Sorption of Olefins by Halogen-Substituted Macrocation-Polyoxometalate Porous Ionic Crystals. Chem. Mater. 2012, 24, 325-330.

49. Katon, J.E.; Kleinlein, R.L. The vibrational spectra and assignments of bromoacetic acid and three of its deuterated analogues. Spectrochim. Acta Part. A 1973, 29, 791-801. [CrossRef]

50. Mathew, S.; Nair, C.G.R. Thermal decomposition kinetics: Part XVII. Kinetics and mechanism of thermal decomposition of bis(ethylenediamine)copper(II) halide monohydrate. Termochim. Acta 1991, 181, 253-268. [CrossRef]

51. Eichloff, R. Uber halogenirte Essigsäuren. Justus Liebigs Ann. Chem. 1905, 342, 115-123.

52. Sheldrick, G.M. SHELXT- Integrated space-group and crystal-structure determination. Acta Crystallogr. Sect. A Found. Adv. 2015, 71, 3-8. [CrossRef]

53. Sheldrick, G.M. Crystal structure refinement with SHELXL. Acta Cryst. 2015, C71, 3-8.

54. Sheldrick, G.M. A short history of SHELX. Acta Cryst. 2008, 64, 112-122. [CrossRef] [PubMed]

55. Prince, E. International Tables for Crystallography, Volume C: Mathematical, Physical and Chemical Tables; Academic Publishers: Dordrecht, The Netherlands, 2004.

56. Georgiev, G.T.; Butler, J.J. Long-term calibration monitoring of Spectralon diffusers BRDF in the air-ultraviolet. Appl. Opt. 2007, 46, 7892-7899. [CrossRef] [PubMed]

57. Macrae, C.F.; Bruno, I.J.; Chisholm, J.A.; Edgington, P.R.; McCabe, P.; Pidcock, E.; Rodriguez-Monge, L.; Taylor, R.; van de Streek, J.; Wood, P.A. Mercury CSD 2.0-New Features for the Visualization and Investigation of Crystal Structures. J. Appl. Cryst. 2008, 41, 466-470. [CrossRef]

58. Frisch, M.J.; Trucks, G.W.; Schlegel, H.B.; Scuseria, G.E.; Robb, M.A.; Cheeseman, J.R.; Scalmani, G.; Barone, V.; Mennucci, B.; Petersson, G.A.; et al. Gaussian 09, Revision, D.01; Gaussian Inc.: Wallingford, CT, USA, 2009.

59. Ditchfield, R.; Hehre, W.J.; Pople, J.A. Self-Consistent Molecular-Orbital Methods. IX. An Extended Gaussian-Type Basis for Molecular-Orbital Studies of Organic Molecules. J. Chem. Phys. 1971, 54, 724-728. [CrossRef]

60. CrystalExplorer17; University of Western Australia: Perth, Australia, 2019.

Sample Availability: Samples of the compounds $\mathbf{1}$ and $\mathbf{2}$ are available from the authors.

Publisher's Note: MDPI stays neutral with regard to jurisdictional claims in published maps and institutional affiliations.

(C) 2020 by the authors. Licensee MDPI, Basel, Switzerland. This article is an open access article distributed under the terms and conditions of the Creative Commons Attribution (CC BY) license (http://creativecommons.org/licenses/by/4.0/). 